\title{
Some theoretical results concerning diphasic flows in thin films
}

\author{
G. Bayada ${ }^{a}$, L. Chupin ${ }^{\mathrm{b}}$, B. Grec ${ }^{\mathrm{c}, *}$ \\ ${ }^{a} I C J$ - UMR 5208, Université Claude Bernard Lyon 1, 43 bd du 11 novembre 1918, \\ F-69622 Villeurbanne Cedex \\ ${ }^{b}$ Laboratoire de Mathématiques, UMR 6620, Université Blaise Pascal, Campus des Cézeaux, \\ F-63177 Aubière Cedex \\ ${ }^{c}$ MAP5 - UMR 8145, Université Paris Descartes,Sorbonne Paris Cité, 45 rue des Saints \\ Pères, F-75720 Paris Cedex 06
}

\begin{abstract}
We are interested in a model for diphasic fluids in thin flows taking into account both the hydrodynamical and the chemical effects at the interface between the two fluids. A limit problem in thin curved channels is introduced heuristically. It is a system coupling the Reynolds equation and the Cahn-Hilliard equation. We study the mathematical properties of this system, and prove an existence result under some smallness condition on the data.
\end{abstract}

Keywords: Cahn-Hilliard equation; Reynolds equation; Thin flow.

\section{Introduction}

In many applications, the geometry of the flow is anisotropic (i.e. one dimension is small with respect to the others), e.g. in lubrication problems. In the Newtonian case, the flow of a fluid between two close surfaces in relative motion is described by an asymptotic approximation of the Navier-Stokes equations, the Reynolds equation. This equation makes it possible to uncouple the pressure and the velocity. Indeed, in thin films, the pressure is considered to be independent of the direction in which the domain is thin. Thus an equation on the pressure only is obtained, and the velocity can be deduced from the pressure. This approach was introduced by Reynolds, and has been rigorously justified in 3 for the Stokes equation, and generalized afterwards in many works: for the steady-case Navier-Stokes equations [1, for the unsteady case [4, for compressible fluids with the perfect gases law [22]... It is of interest to investigate how this approach can be used for the case of a two fluid flow.

\footnotetext{
* Corresponding author

Email addresses: guy.bayada@insa-lyon.fr (G. Bayada), laurent.chupin@math.univ-bpclermont.fr (L. Chupin), berenice.grec@parisdescartes.fr (B. Grec)
} 
A first diphasic model consists in introducing a variable viscosity $\eta$, which is either equal to the viscosity $\eta_{1}$ of one fluid or the viscosity $\eta_{2}$ of the other fluid (that is to say that the fluids are considered to be non-miscible). The behavior of $\eta$ is described by a transport equation. In that case, when assuming the interface between the two fluids to be the graph of a function, the asymptotic equations corresponding to the thin film approximation can be interpreted as a generalized Buckley-Leverett equation, which governs the behavior of the saturation (i.e. the proportion of one fluid in the mixture) inside the gap, coupled with a generalized Reynolds equation, which governs the behavior of the pressure. These equations are investigated in 24] without shear effects, and in [7, 14] with shear effects. One of the main disadvantages of the method is that the fluid interface is supposed to be the graph of a function, which hinders for example the formation of bubbles. In addition, this kind of model only takes into account hydrodynamical effects between the two phases, and surface tension effects are neglected.

The second class of models describing diphasic flows, which has been used up to now only for the Navier-Stokes equations, is the class of the so-called diffuse interface models. They take into account chemical properties at the interface between the two fluids, enabling an exchange between the two phases. In this paper, we use a Cahn-Hilliard equation, which involves an interaction potential, enhanced with a transport term. Thus this model describes both the chemical and the hydrodynamical properties of the flow. An order parameter $\varphi$ is introduced, for example the volumic fraction of one phase in the mixture. The surface tension can be taken into account via an additional term depending on $\varphi$ in the Navier-Stokes equations. This kind of model has been studied for the complete Navier-Stokes equations in [8, and for viscoelastic fluids in [12.

In this paper, we consider an asymptotic system (i.e. a thin film approximation) for a diphasic fluid modelled by the Cahn-Hilliard equation. In a similar way as for the Newtonian case, the Navier-Stokes equations are approximated by a modified Reynolds equation, in which the viscosity is not constant anymore. We study the Reynolds/Cahn-Hilliard system, and prove the existence and the regularity of a weak solution under a smallness assumption on the initial data and the geometry.

Let us describe briefly the main steps of the mathematical analysis. First, we study the Reynolds equation and investigate the regularity of the pressure and the velocity as functions of the order parameter. Next, we prove the existence of a solution to the system Reynolds/Cahn-Hilliard, by using a Galerkin process, which consists in introducing finite dimension approximations of $\varphi$. After obtaining a priori estimates for these approximations, we conclude that they converge to a solution of the system Reynolds/Cahn-Hilliard.

This paper is organized as follows. In Section 2 we introduce the twodimensional model for a diphasic fluid in a thin film, which consists of a generalized Reynolds equation and of a diffuse-interface model (the Cahn-Hilliard equation). In Section 3 , we state the main theorem, and give the main steps and difficulties of the proof. In Section 4 we deal with the Reynolds equation, and obtain some existence and regularity result on the velocity field and the pres- 
sure. In Section 5, we first introduce some specific results on trace estimates and Poincaré inequalities. They are used in the rest of the section for obtaining a priori estimates for the Cahn-Hilliard equation. At last, convergence results are deduced from these estimates, and allow to conclude the proof of the main theorem. Section 6 presents some preliminary numerical results obtained with this model in order to highlight the features of the model.

\section{Modelling a diphasic fluid in a thin film}

In this section, we will first present how a fluid is described in a thin domain by the Reynolds equation. Next, we introduce the hydrodynamical CahnHilliard model for any fluid. Lastly, we combine both aspects and state the model of a diphasic fluid in a thin domain.

We introduce the physical domain $\bar{\Omega}$

$$
\bar{\Omega}=\left\{(\bar{x}, \bar{z}) \in \mathbb{R}^{2}, 0<\bar{x}<L, 0<\bar{z}<h(x)\right\} .
$$

The thin film approximation for an incompressible fluid leads to the following equations (see [3]), describing the behavior of the pressure $p$ and the velocity field $\boldsymbol{u}=(u, v), \eta$ being the viscosity of the fluid.

$$
\partial_{\bar{z}}\left(\eta \partial_{\bar{z}} u\right)=\partial_{\bar{x}} p, \quad \partial_{\bar{z}} p=0, \quad \partial_{\bar{x}} u+\partial_{\bar{z}} v=0 .
$$

In these equations, the thin film assumption leads to the decoupling of the pressure and the velocity, as well as the simplification of the equations.

We will see that it is possible to prove an existence theorem assuming a small size condition on the physical domain $\bar{\Omega}$ (see Theorem 3.3 ). In order to understand the dependance of the solution with respect to the domain $\bar{\Omega}$, we rescale the spatial variable $(\bar{x}, \bar{z})$ using a dilatation coefficient $\lambda$. More precisely, we suppose that the domain is small and can be written as

$$
\bar{\Omega}=\left\{(\bar{x}, \bar{z}) \in \mathbb{R}^{2}, 0<\lambda \bar{x}<\lambda L, 0<\lambda \bar{z}<\lambda h(x)\right\},
$$

and we rewrite the system using the following change of variable and domain

$$
\lambda \bar{x} \rightarrow x, \quad \lambda \bar{z} \rightarrow z, \quad \bar{\Omega} \rightarrow \Omega=\left\{(x, z) \in \mathbb{R}^{2}, 0<x<L, 0<z<h(x)\right\} .
$$

We assume that there exists three constants $\left(h_{m}, h_{M}, h_{M}^{\prime}\right) \in \mathbb{R}_{+}^{3}$ such that the function $h \in \mathcal{C}^{2}(\mathbb{R})$ (see Fig. 1) satisfies

$$
\forall x \in[0, L], \quad 0<h_{m} \leqslant h(x) \leqslant h_{M} \quad \text { and } \quad\left|h^{\prime}(x)\right| \leqslant h_{M}^{\prime},
$$

and $h^{\prime}(L)=0$ as well as

$$
\exists \tilde{\varepsilon}>0 \text { such that } \forall x \in[0, \tilde{\varepsilon}], \quad h^{\prime}(x)=h^{\prime \prime}(x)=0,
$$

Observe that the regularity of $h$ ensures that the domain $\Omega$ defined by (2) satisfies the segment property and cone property (see [2, $\S 4.2$ and 4.3]). 


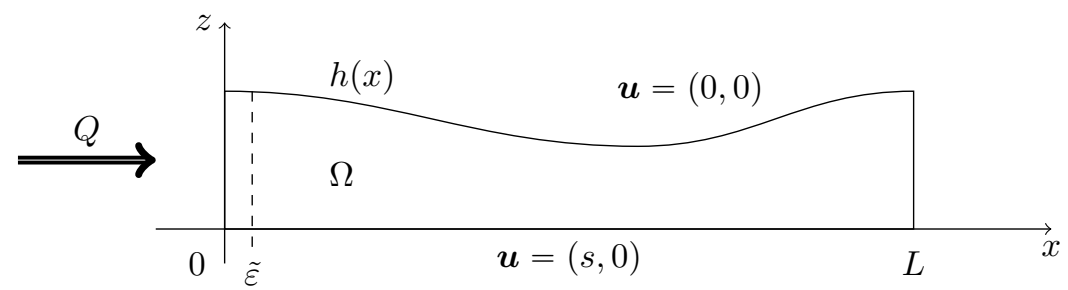

Figure 1: Domain $\Omega$ and boundary conditions on the velocity

The Reynolds equation now writes

$$
\partial_{z}\left(\eta \partial_{z} u\right)=\lambda \partial_{x} p, \quad \partial_{z} p=0, \quad \partial_{x} u+\partial_{z} v=0 .
$$

We choose boundary conditions on $\boldsymbol{u}$ suitable for lubrication applications: Dirichlet boundary conditions are imposed on the velocity on $\{z=0\}$ and $\{z=h(x)\}$ in order to model shear effects. The boundary conditions are written:

$$
\forall x \in[0, L] \quad u(x, 0)=s \quad \text { and } \quad u(x, h(x))=v(x, 0)=v(x, h(x))=0 .
$$

Without loss of generality, the constant shear velocity $s$ is supposed to be positive. For the lateral part of the boundary, it has been showed in 3 that only the input flow $Q=\int_{0}^{h(0)} u(0, \xi) d \xi$ needs to be prescribed. Observe that according to the divergence-free condition and the boundary conditions on $\boldsymbol{u}$, this flow is constant on any "vertical" section of the domain:

$$
\begin{aligned}
\partial_{x}\left(\int_{0}^{h(x)} u(x, \xi) d \xi\right) & =\underbrace{h^{\prime}(x) u(x, h(x))}_{=0}+\int_{0}^{h(x)} \partial_{x} u(x, \xi) d \xi=-\int_{0}^{h(x)} \partial_{\xi} v(x, \xi) d \xi \\
& =-v(x, h(x))+v(x, 0)=0,
\end{aligned}
$$

thus

$$
Q=\lambda \int_{0}^{h(x)} u(x, \xi) d \xi, \quad \forall x \in(0, L) .
$$

Remark 2.1. We use the Reynolds equation to describe the behavior of the fluid. This equation is an approximation of the (Navier)-Stokes system for thin domains (in which the height is much smaller than the length). The anisotropy of the physical domain is therefore taken into account in this step. Further, the equation is written down in (4) in a rescaled form in the domain $\Omega$ (with length and height of the same order of magnitude). No assumption on the shape is needed for the domain $\Omega$. As we already stated, the parameter $\lambda$ will allow us to control the smallness of the physical domain.

\subsection{Modelling one fluid in a thin domain}

The usual procedure [3] is to integrate twice the first equation of (4) with respect to $z$, make use of the boundary conditions (5) and of the fact that $\partial_{z} p=0$. This 
allows us to express $u$ as a function of $p$ :

$$
u(x, z)=\frac{z(z-h(x))}{2 \eta} \lambda \partial_{x} p(x)+s\left(1-\frac{z}{h(x)}\right) .
$$

Then, putting this expression in the divergence-free equation leads to the Reynolds equation:

$$
\lambda \partial_{x}\left(\frac{h^{3}}{12 \eta} \partial_{x} p\right)=s \partial_{x}\left(\frac{h}{2}\right) .
$$

A first boundary condition on $p$ is deduced from the ones on $\boldsymbol{u}$. In fact, the choice of the input flow $Q$ corresponds to a Neumann condition for $p$ at $x=0$. This condition can be determined as a function of $Q$ by

$$
Q=\lambda \int_{0}^{h(0)} u(0, \xi) d \xi=-\lambda^{2} \partial_{x} p(0) \frac{h(0)^{3}}{12 \eta}+\frac{\lambda s h(0)}{2} .
$$

Let us denote $w:=\frac{12 \eta(\lambda \operatorname{sh}(0) / 2-Q)}{\lambda^{2} h(0)^{3}}=\partial_{x} p(0)$.

Moreover, the solution $p$ of (8) with the Neumann boundary condition $\partial_{x} p(0)=w$ is defined up to a constant. We can thus choose $p(L)=0$ to gain a well-defined pressure $p$. It is to be noticed that once $p$ is computed from (8), then (7) allows us to compute $u$, while the other component $v$ of the velocity field is obtained by:

$$
v(x, z)=-\int_{0}^{z} \partial_{x} u(x, \xi) d \xi
$$

\subsection{Modelling a mixture}

Since we want to study the mixture of two fluids, we introduce an order parameter $\varphi$ describing the volumic fraction of one fluid in the flow. All physical parameters can be written as functions of $\varphi$, in particular the viscosity $\eta$. We assume that the function $\eta$ satisfies $\eta \in \mathcal{C}^{1}(\mathbb{R})$ such that

$$
\exists\left(\eta_{m}, \eta_{M}, \eta_{M}^{\prime}\right) \in \mathbb{R}^{3} ; \forall \varphi \in \mathbb{R}, \quad 0<\eta_{m} \leqslant \eta(\varphi) \leqslant \eta_{M} \quad \text { and } \quad \eta^{\prime}(\varphi) \leqslant \eta_{M}^{\prime} .
$$

A possible explicit form of the viscosity is given in the following Remark:

Remark 2.2. For $\varphi \in[-1,1]$, we can use a specific realistic law as a function of the viscosities of the two fluids $\eta_{1}$ and $\eta_{2}$ (see [10] or [23]):

$$
\frac{1}{\eta(\varphi)}=\frac{1+\varphi}{2 \eta_{1}}+\frac{1-\varphi}{2 \eta_{2}} \quad \text { for } \varphi \in[-1,1]
$$

so that $\varphi=1$ and $\varphi=-1$ correspond respectively to the fluids of viscosity $\eta_{1}$ and $\eta_{2}$ only. However, we will not always be able to prove mathematically that $\varphi$ remains in the interval $[-1,1]$ (see [8]). 
The effects of a possible variation of the density in the mixture will not be taken into account in this paper. Therefore, the density of the mixture is assumed to be constant (i.e. the two densities of the two incompressible phases $\rho_{1}$ and $\rho_{2}$ are supposed to be equal). Let us notice that due to the loss of the local conservation equation for the density, the non-homogeneous case $\rho_{1} \neq \rho_{2}$ induces further difficulties (see [9]).

We choose the Cahn-Hilliard equation in order to describe the evolution of $\varphi$. This equation consists of both a transport term, taking the mechanical effects into account, and a diffusive term modelling the chemical effects. The CahnHilliard equation is written in the rescaled domain $\Omega$ :

$$
\begin{aligned}
& \lambda \partial_{t} \varphi+\boldsymbol{u} \cdot \nabla \varphi-\frac{1}{\lambda \mathcal{P} e} \operatorname{div}(\mathcal{B}(\varphi) \nabla \mu)=0, \\
& \mu=-\frac{\alpha^{2}}{\lambda^{2}} \Delta \varphi+F^{\prime}(\varphi) .
\end{aligned}
$$

Recall that the constant $\lambda$ is a rescaling constant allowing us to follow the dependance on the domain size. The variable $\mu$ is the chemical potential, $\mathcal{B}(\varphi)$ is called mobility, $\mathcal{P} e$ is the Péclet number, $\alpha$ is a non-dimensional parameter measuring the thickness of the diffuse interface, and the function $F$ is called Cahn-Hilliard potential. Physical considerations show that $F$ must have a double-well structure, each of the wells representing one of the two fluids. A rational choice for $F$ is given by a logarithmic form (for more details, we refer to [16] or [20])

$$
F(\xi)=1-\xi^{2}+c((1+\xi) \log (1+\xi)+(1-\xi) \log (1-\xi)),
$$

for some constant $0<c<1$, or its polynomial approximation

$$
F(\xi)=\left(1-c^{\prime} \xi^{2}\right)^{2},
$$

where $c^{\prime}$ is another constant. These physically realistic potentials share several mathematical properties. In the following, we prove mathematical results for potentials $F$ having these properties:

- The function $F$ is supposed to be regular (e.g. of class $\mathcal{C}^{2}(\mathbb{R})$ ).

- Since $F$ is a physical potential, it is bounded from below. Moreover, only the derivative of $F$ occurs in the equations, therefore the addition of a constant does not change the equations. It is thus realistic to make the following assumption:

$$
\exists F_{0}>0 ; \forall \xi \in \mathbb{R} \quad F(\xi) \geqslant F_{0} .
$$

- The convexity of the potential corresponds to the stability of the mixture. Usual potentials contain some stable and unstable regions (see for example Figure 2p. In order to include such cases, we impose:

$$
\exists F_{5} \geqslant 0 ; \forall \xi \in \mathbb{R} \quad F^{\prime \prime}(\xi) \geqslant-F_{5} .
$$


- Moreover, the following hypothesis on the growth of the potential is imposed:

$$
\begin{gathered}
\exists F_{1}, F_{2}>0 \quad \exists r>1 ; \forall \xi \in \mathbb{R} \\
\left|F^{\prime}(\xi)\right| \leqslant F_{1}|\xi|^{r}+F_{2} \quad \text { and } \quad\left|F^{\prime \prime}(\xi)\right| \leqslant F_{1}|\xi|^{r-1}+F_{2} .
\end{gathered}
$$

This hypothesis is satisfied for any polynomial function.

- At last, we state a generalization of the convexity:

$$
\begin{array}{ll}
\forall \gamma \in \mathbb{R} \quad \exists F_{3}(\gamma)>0, F_{4}(\gamma) \geqslant 0 ; \\
\forall \xi \in \mathbb{R} \quad(\xi-\gamma) F^{\prime}(\xi) \geqslant F_{3}(\gamma) F(\xi)-F_{4}(\gamma) .
\end{array}
$$

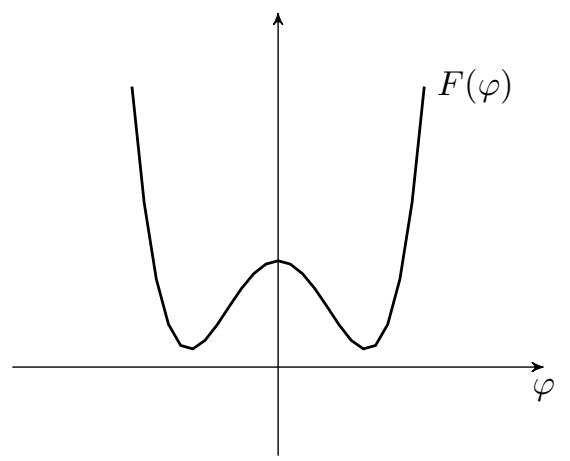

Figure 2: Possible shape of the potential $F(\varphi)$

These assumptions are satisfied by a function of the form $F(\varphi)=\frac{\varphi^{4}}{4}-\frac{\varphi^{2}}{2}+F_{0}$ (as in Figure 2), which can be used as a model case.

As far as the mobility $\mathcal{B}$ is concerned, it is supposed to be regular $\mathcal{B} \in \mathcal{C}^{2}(\mathbb{R})$, positive, and bounded from above and from below:

$$
\exists\left(B_{m}, B_{M}\right) \in \mathbb{R}^{2} ; \forall \xi \in \mathbb{R} \quad 0<\mathcal{B}_{m} \leqslant \mathcal{B}(\xi) \leqslant \mathcal{B}_{M} .
$$

Let us mention that other types of functions $\mathcal{B}$ can be considered, in particular the degenerate case $\mathcal{B}(\xi)=\left(1-\xi^{2}\right)^{\sigma}$, with $\sigma \geqslant 0$, which has been studied in [8] and in [17, but introduces further mathematical difficulties.

Equations (11)-12 must be equipped with boundary conditions on $\varphi$ and $\mu$. We are interested here in injection phenomena, which arise for example in lubrication or polymer injection problems. To this end, it is important to control the composition of the input. Thus we use Dirichlet boundary conditions on some part of the boundary, namely where the fluid is supplied. For the other part of the boundary, classical Neumann boundary conditions for both $\varphi$ and $\mu$ are considered. Let us observe that in previous works ([8] and [12]) Neumann boundary conditions were imposed on the whole boundary. 


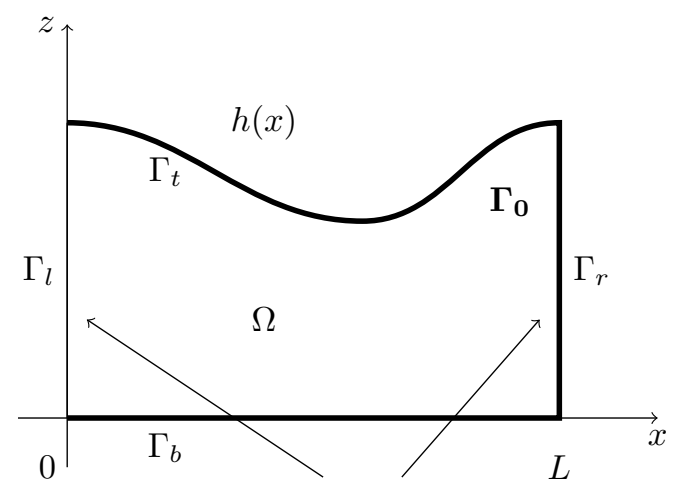

Figure 3: Domain $\Omega$ and notations for the boundary

Let us define (see Figure 3)

$$
\begin{array}{ll}
\Gamma_{l}=\left\{(0, z) \in \mathbb{R}^{2},\right. & 0<z<h(0)\}, \quad \Gamma_{r}=\left\{(L, z) \in \mathbb{R}^{2}, \quad 0<z<h(L)\right\}, \\
\Gamma_{b}=\left\{(x, 0) \in \mathbb{R}^{2},\right. & 0<x<L\}, \quad \Gamma_{t}=\left\{(x, z) \in \mathbb{R}^{2}, \quad z=h(x)\right\}, \\
& \Gamma_{0}=\{(x, z) \in \partial \Omega, \quad x>0\} .
\end{array}
$$

Thus, the boundary conditions are written, denoting $\boldsymbol{n}$ the exterior normal to the domain, as follows:

$$
\left.\varphi\right|_{\Gamma_{l}}=\varphi_{l},\left.\quad \mu\right|_{\Gamma_{l}}=0 \quad \text { and }\left.\quad \frac{\partial \varphi}{\partial \boldsymbol{n}}\right|_{\Gamma_{0}}=0,\left.\quad \frac{\partial \mu}{\partial \boldsymbol{n}}\right|_{\Gamma_{0}}=0
$$

for some given boundary value $\varphi_{l}$ defined on $\Gamma_{l}$, satisfying the following hypothesis:

Hypothesis 2.3. We assume that $\varphi_{l} \in H^{7 / 2}\left(\Gamma_{l}\right)$ satisfies

$$
\varphi_{l}^{\prime}(0)=\varphi_{l}^{\prime}(h(0))=0, \quad\left|\varphi_{l}^{\prime}\right|_{L^{2}\left(\Gamma_{l}\right)}<\bar{\varepsilon} .
$$

for some small $\bar{\varepsilon}>0$ depending on all the data. We will explain further how $\bar{\varepsilon}$ is determined (see Proposition 5.12).

Finally, let us define the initial condition: $\left.\varphi\right|_{t=0}=\varphi_{0} \in H^{3}(\Omega)$, where $\varphi_{0}$ is supposed to be satisfying the same boundary conditions as $\varphi$. Compatibility conditions also imply that $\mu_{0}$ defined by $\mu_{0}=-\frac{\alpha^{2}}{\lambda^{2}} \Delta \varphi_{0}+F^{\prime}\left(\varphi_{0}\right)$ satisfies the same boundary conditions as $\mu$.

\subsection{Modelling a mixture in thin films}

A diphasic flow in a thin domain is described by a modified Reynolds system of the form (4), where the viscosity $\eta$ is not constant anymore but depends on the order parameter $\varphi$. Because of the non-constant viscosity, the coefficients 
in the Reynolds equation (which depend on $\eta$ ) depend on $\varphi$. Let us introduce the following expressions that will be useful in the following:

$$
a(x, z)=\int_{0}^{z} \frac{d \xi}{\eta(\varphi(x, \xi))}, \quad b(x, z)=\int_{0}^{z} \frac{\xi d \xi}{\eta(\varphi(x, \xi))}, \quad c(x, z)=\int_{0}^{z} \frac{\xi^{2} d \xi}{\eta(\varphi(x, \xi))},
$$

and

$$
\widetilde{a}(x)=a(x, h(x)), \quad \widetilde{b}(x)=b(x, h(x)), \quad \widetilde{c}(x)=c(x, h(x)),
$$

for all $(x, z) \in \Omega$. We also define:

$$
\widetilde{d}(x)=\widetilde{c}(x)-\frac{\widetilde{b}(x)^{2}}{\widetilde{a}(x)} \quad \text { and } \quad \widetilde{e}(x)=\frac{\widetilde{b}(x)}{\widetilde{a}(x)} .
$$

Following the same procedure as in Section 2.1. we integrate twice the first equation of (4) with non-constant viscosity and using the boundary conditions, we obtain for all $(x, z) \in \Omega$ :

$$
\begin{aligned}
& u(x, z)=\left(b(x, z)-\frac{\widetilde{b}(x)}{\widetilde{a}(x)} a(x, z)\right) \lambda \partial_{x} p(x)+\left(1-\frac{a(x, z)}{\widetilde{a}(x)}\right) s, \\
& v(x, z)=-\int_{0}^{z} \partial_{x} u(x, \xi) d \xi .
\end{aligned}
$$

We use the fact that $\boldsymbol{u}$ is divergence-free and the boundary conditions in order to write

$$
\int_{0}^{h(x)} \partial_{x} u(x, z) d z=\partial_{x}\left(\int_{0}^{h(x)} u(x, z) d z\right)=0 .
$$

After integrating 22 , we obtain

$$
\lambda \partial_{x}\left(\widetilde{d}(x) \partial_{x} p(x)\right)=s \partial_{x}(\widetilde{e}(x)),
$$

where the coefficients $\widetilde{d}$ and $\tilde{e}$ are given by 21 . Therefore the whole system (Reynolds/ Cahn-Hilliard) is written:

$$
\left\{\begin{array}{l}
\lambda \partial_{x}\left(\tilde{d} \partial_{x} p\right)=s \partial_{x} \widetilde{e} \\
u=\left(b-\frac{a \tilde{b}}{\widetilde{a}}\right) \lambda \partial_{x} p+s\left(1-\frac{a}{\widetilde{a}}\right) \\
v(\cdot, z)=-\int_{0}^{z} \partial_{x} u(\cdot, \xi) d \xi \\
\lambda \partial_{t} \varphi+u \partial_{x} \varphi+v \partial_{z} \varphi-\frac{1}{\lambda \mathcal{P} e} \operatorname{div}(\mathcal{B}(\varphi) \nabla \mu)=0 \\
\mu=-\frac{\alpha^{2}}{\lambda^{2}} \Delta \varphi+F^{\prime}(\varphi) .
\end{array}\right.
$$

The coefficients $a, b, \widetilde{a}, \widetilde{b}, \widetilde{d}, \widetilde{e}$ are explicit functions of $\varphi$ (given by (20)-(21)). The functions $\mathcal{B}, F$ are also known functions of $\varphi$. The quantities $\mathcal{P} e, \alpha$ are 
physical constants. The boundary conditions on $\varphi$ and $\mu$ are given in (18). Let us notice that equations (26b)-(26c) imply that the following boundary conditions are satisfied for $\boldsymbol{u}$ :

$$
\begin{aligned}
& u(x, 0)=s, \quad u(x, h(x))=v(x, 0)=v(x, h(x))=0, \\
& \lambda \int_{0}^{h(0)} u(0, \xi) d \xi=Q .
\end{aligned}
$$

As far as the pressure $p$ is concerned, we impose an homogeneous Dirichlet boundary condition at $x=L$ and a Neumann boundary condition at $x=0$, which is given as a function of $Q$ and $s$. These boundary conditions are written:

$$
w:=\partial_{x} p(0)=\frac{Q-s \lambda\left(h(0)-\frac{1}{\widetilde{a}(0)} \int_{0}^{h(0)} a(0, \xi) d \xi\right)}{\lambda^{2}\left(\int_{0}^{h(0)} b(0, \xi) d \xi-\frac{\widetilde{b}(0)}{\widetilde{a}(0)} \int_{0}^{h(0)} a(0, \xi) d \xi\right)}, \quad p(L)=0 .
$$

\section{Statement of the main result}

\subsection{Main theorem}

Notations 3.1. Let us define some notations and function spaces.

(i) For the usual Sobolev spaces, we denote by $|\cdot|_{p}$ the $L^{p}$-norm in $\Omega$, and by $\|\cdot\|_{s}$ the $H^{s}$-norm in $\Omega$. We also introduce $\|\cdot\|_{2}$ which contains the second-order derivatives:

$$
\|\| \cdot \|_{2}^{2}=\left|\partial_{z} \cdot\right|_{2}^{2}+\left|\partial_{x}^{2} \cdot\right|_{2}^{2}+\left|\partial_{x z}^{2} \cdot\right|_{2}^{2}+\left|\partial_{z}^{2} \cdot\right|_{2}^{2} .
$$

(ii) Let us define the following function spaces:

$$
\begin{aligned}
\mathcal{X}(\Omega) & =\left\{f \in H^{1}(\Omega) \cap L^{\infty}(\Omega), \quad \partial_{z} f \in H^{1}(\Omega)\right\}, \\
\Phi_{0}^{1} & =\overline{\left\{\phi \in \mathcal{D}(\bar{\Omega}),\left.\quad \phi\right|_{\Gamma_{l}}=0\right\}} H^{1}(\Omega)
\end{aligned}
$$

We introduce the weak form of 26.

Problem 3.2. Let $\varphi_{l} \in H^{7 / 2}\left(\Gamma_{l}\right)$, and $\varphi_{0} \in H^{3}(\Omega)$ satisfying (18), and $T>0$. Find $(p, u, v, \varphi, \mu)$ such that

- the following regularity is satisfied:

$$
\begin{gathered}
p \in L^{\infty}\left(0, T ; H^{2}(0, L)\right), \quad u \in L^{\infty}(0, T ; \mathcal{X}(\Omega)), \quad v \in L^{\infty}\left(0, T ; L^{2}(\Omega)\right), \\
\varphi \in L^{\infty}\left(0, T ; H^{1}(\Omega)\right) \cap L_{l o c}^{2}\left(0, T ; H^{3}(\Omega)\right) \cap \mathcal{C}^{0}\left((0, T) ; H^{1}(\Omega)\right), \\
\mu \in L_{l o c}^{2}\left(0, T ; \Phi_{0}^{1}\right) .
\end{gathered}
$$


- the velocity field $\boldsymbol{u}_{\varphi}=(u, v)$ is given as a function of $\varphi$ by (26a), 26b), (26c) equipped with the boundary conditions (27), 28) and 291.

- for any $\psi \in \Phi_{0}^{1}$,

$$
\lambda \int_{\Omega} \partial_{t} \varphi \psi+\int_{\Omega} \frac{1}{\lambda \mathcal{P} e} \mathcal{B}(\varphi) \nabla \mu \nabla \psi+\int_{\Omega} \boldsymbol{u}_{\varphi} \cdot \nabla \varphi \psi=0,
$$

with

$$
\mu=-\frac{\alpha^{2}}{\lambda^{2}} \Delta \varphi+F^{\prime}(\varphi)
$$

- the initial condition $\left.\varphi\right|_{t=0}=\varphi_{0}$ is satisfied as well as the boundary conditions (18) for $\varphi$.

The following sections are dedicated to the proof of the main theorem:

Theorem 3.3. Let $T>0, \varphi_{l}$ satisfying Hypothesis (2.3), $\varphi_{0} \in H^{3}(\Omega)$ satisfying (18), $F$ and $\eta$ satisfying the assumptions stated in Section 2.2. If $\lambda$ is small enough then there exists a solution $(p, u, v, \varphi, \mu)$ to Problem 3.2.

\subsection{Sketch of the proof}

We present here the sketch of the proof of the main theorem. All the details and computations are given in Sections 4 and 5. The proof is divided into two main parts, since the Reynolds equation and the Cahn-Hilliard are treated separately.

Step 1. As far as the Reynolds equation is concerned, we prove the following proposition:

Proposition 3.4. Assume that the viscosity $\eta$ satisfies (9). For any $\varphi \in H^{1}(\Omega)$, the Reynolds equation 26a) equipped with the boundary conditions 29) admits a unique solution which satisfies

$$
\partial_{x} p \in H^{1}(0, L) .
$$

The velocity field $\boldsymbol{u}=(u, v)$ given as a function of $p$ by (26b)-(26c) satisfies

$$
u \in H^{1}(\Omega) \cap L^{\infty}(\Omega) \quad \text { and } v \in L^{2}(\Omega) \text {, with } \partial_{z} v \in L^{2}(\Omega) .
$$

Moreover, we have the following estimates

$$
|u|_{\infty} \leqslant C \quad \text { and } \quad|v|_{2} \leqslant C\|\varphi\|_{1},
$$

where the constant $C$ does not depend on the scaling defined by $\lambda$.

Let us sketch the main steps of the proof of Proposition 3.4 . 
- The Reynolds equation can be solved explicitly, so that $p$ is given as a function of the coefficients $\widetilde{d}$ and $\tilde{e}$ (given as functions of $\varphi$ by (21)): recalling definition (29) of $w$, we can integrate the Reynolds equation once and obtain

$$
\lambda \widetilde{d} \partial_{x} p=s \tilde{e}+\lambda \widetilde{d}(0) w-s \widetilde{e}(0),
$$

where the coefficients $\widetilde{d}(0)$ and $\widetilde{e}(0)$ only depend on $\varphi_{l}$ and are thus known. If $\widetilde{d}$ does not vanish, we compute formally $\partial_{x} p$, and then $p$ using the boundary condition $p(L)=0$. In order to obtain estimates on the pressure, we have to prove that the coefficients $\widetilde{d}$ and $\tilde{e}$ are regular enough (see Lemma 4.1), and that $\widetilde{d}(\varphi)$ is greater than a strictly positive constant (i.e. the operator $\partial_{x}\left(d \partial_{x} \cdot\right)$ must be coercive, see Lemma 4.2.

- As far as the velocity is concerned, $u$ is given by

$$
u=\lambda f \partial_{x} p+g,
$$

where the coefficients are given by $f=\left(b-\frac{\tilde{b}}{\widetilde{a}} a\right)$ and $g=\left(1-\frac{a}{\widetilde{a}}\right) s$ (and $a, b, \widetilde{a}, \tilde{b}$ are defined in (20)). It is enough to prove the regularity of $f$ and $g$ in order to deduce the needed estimate on $u$ from the estimate on $\partial_{x} p$ (see Lemma 4.3 ).

- The velocity $v$ is given by

$$
v(x, z)=-\int_{0}^{z} \partial_{x} u(x, \xi) d \xi,
$$

and the regularity of $v$ follows from the regularity of $u$ (see Lemma 4.4).

Step 2. As far as the Cahn-Hilliard equation is concerned, we proceed as in the earlier works on Cahn-Hilliard equation (e.g. [8]), and we apply the Galerkin method in order to prove the existence of a solution to the system (30)-(31). This process consists in building approximate solutions $\left(\varphi_{n}, \mu_{n}\right)$ in finite dimension (see Section 5.2), for which the existence follows from the Cauchy-Lipschitz theorem. For these approximate solutions $\left(\varphi_{n}, \mu_{n}\right)$, we prove the following proposition (see Section 5.5):

Proposition 3.5. For all $0 \leqslant t \leqslant T$, let

$$
\begin{aligned}
& \mathcal{Y}(t)=\frac{\alpha^{2}}{2 \lambda^{2}}\left|\nabla \varphi_{n}(t)\right|_{2}^{2}+\int_{\Omega} F\left(\varphi_{n}(t)\right), \\
& \mathcal{Z}(t)=\frac{\alpha^{2}}{2 \lambda^{2}}\left|\nabla \varphi_{n}(t)\right|_{2}^{2}+\left|\nabla \mu_{n}(t)\right|_{2}^{2}+\left|\Delta \varphi_{n}(t)\right|_{2}^{2}+\int_{\Omega} F\left(\varphi_{n}(t)\right) .
\end{aligned}
$$

Then the following estimate is satisfied:

$$
\mathcal{Y}^{\prime}(t)+C_{1} \mathcal{Z}(t) \leqslant f(\mathcal{Y}(t)) \mathcal{Z}(t)+C_{2}
$$

where $C_{1}, C_{2}$ are positive constants, and $f: \mathbb{R} \rightarrow \mathbb{R}$ is a continuous function satisfying $f(0)=0$. 
Let us emphasize the main features of the proof:

- Although estimates on the Cahn-Hilliard equation are similar to the ones in 8 or [13, they involve supplementary terms due to the different boundary conditions: because of the non-homogeneous Dirichlet condition on $\varphi_{n}$ on the left-hand side of the domain (fluid injection), the conservation of the quantity of each fluid is not satisfied anymore (in the sense that the mean value $m\left(\varphi_{n}\right)=\frac{1}{|\Omega|} \int_{\Omega} \varphi_{n}$ is not constant with respect to time). For example, since $m\left(\varphi_{n}\right)$ is not constant, we cannot apply classical inequalities on $\varphi_{n}-m\left(\varphi_{n}\right)$, such as the Poincaré inequality, and we have to work with the boundary value of $\varphi_{n}$ on the left-hand side of the domain (see Sections 5.3 and 5.4 .

- In order to control the boundary and source terms with the ones on the left-hand side of the estimate, we have to work in adequate function spaces and choose in a suitable way the coefficients in front of each term. This is obtained only by imposing a smallness assumption on $\lambda$ which depends on all other data of the problem.

From Proposition 3.5, we deduce the convergence of the linear terms. However, it is not enough to conclude the convergence of the nonlinear terms and the initial condition. To this end, we need more regularity on $\varphi_{n}$ and will prove the following proposition:

Proposition 3.6. There exists $C>0$ such that for any $T>0$ :

$$
\left\|\varphi_{n}\right\|_{L^{2}\left(0, T ; H^{3}(\Omega)\right)} \leqslant C T+C, \quad\left\|\frac{d \varphi_{n}}{d t}\right\|_{L^{2}\left(0, T ; H^{-1}(\Omega)\right)} \leqslant C T+C .
$$

This proposition allows us to deduce the convergence of all terms in adequate function spaces, using classical compacity results from [25].

\section{About the Reynolds equation}

The letter $C$ will then denote any constant depending on physical parameters $\left(s, Q, \eta_{M}, \eta_{m}, \mathcal{P} e, \alpha, F_{1}, F_{2}, F_{3}, F_{4}, \mathcal{B}_{m}, L, h(x), \ldots\right)$, but independent of the unknowns $(\boldsymbol{u}, p, \varphi, \mu)$ and of $\lambda$.

\subsection{Regularity of the coefficients}

Lemma 4.1. Assume that the viscosity $\eta$ satisfies (9). If $\varphi \in H^{1}(\Omega)$, the coefficients defined in (20), (21) have the following regularity:

$$
a, b, c \in \mathcal{X}(\Omega),
$$

$\widetilde{a}, \widetilde{b}, \widetilde{c}, \widetilde{d}, \widetilde{e} \in H^{1}(0, L)$. 
Proof. Assume $\varphi \in H^{1}(\Omega)$. The terms $a, b, c$ are of the form $\int_{0}^{z} \xi^{i} / \eta(\varphi(x, \xi)) d \xi$, for $i=0,1,2$ (see definition (20) of $a, b, c$ ). We will present the details of the proof for the case $i=1$. The same computations can be used to obtain the regularity results for $i=0, i=2$. Let

$$
b(x, z)=\int_{0}^{z} \frac{\xi}{\eta(\varphi(x, \xi))} d \xi .
$$

Let us prove that $b \in \mathcal{X}(\Omega)$ for any $\varphi \in H^{1}(\Omega)$.

$\triangleright$ First we prove that $b \in L^{2}(\Omega):$ for any $(x, z) \in \Omega$, we have

$$
b(x, z)^{2}=\left(\int_{0}^{z} \frac{\xi}{\eta(\varphi(x, \xi))} d \xi\right)^{2} \leqslant\left(\frac{1}{\eta_{m}} \int_{0}^{z} \xi d \xi\right)^{2} \leqslant C z^{4} .
$$

After integrating with respect to $z$ and $x$, we get

$$
\int_{0}^{L} \int_{0}^{h(x)} b(x, z)^{2} d z d x \leqslant C .
$$

$\triangleright$ Next, we show that $b \in H^{1}(\Omega)$ and $\partial_{z} b \in H^{1}(\Omega)$ :

- On one hand,

$$
\partial_{x} b(x, z)=-\int_{0}^{z} \frac{\xi \eta^{\prime}(\varphi(x, \xi))}{\eta(\varphi(x, \xi))^{2}} \partial_{x} \varphi(x, \xi) d \xi,
$$

with $\partial_{x} \varphi \in L^{2}(\Omega)$. Let $(x, z) \in \Omega$. Using hypothesis (9), we compute

$$
\begin{aligned}
\left|\partial_{x} b(x, z)\right|^{2} & =\left(\int_{0}^{z} \frac{\xi \eta^{\prime}(\varphi(x, \xi))}{\eta(\varphi(x, \xi))} \partial_{x} \varphi(x, \xi) d \xi\right)^{2} \\
& \leqslant \frac{\eta_{M}^{\prime 2}}{\eta_{m}^{2}} \int_{0}^{z} \xi^{2} d \xi \int_{0}^{z}\left|\partial_{x} \varphi(x, \xi)\right|^{2} d \xi \leqslant C z^{3} \int_{0}^{h(x)}\left|\partial_{x} \varphi(x, \xi)\right|^{2} d \xi
\end{aligned}
$$

After integrating with respect to $z$ and then with respect to $x$, we get

$$
\begin{aligned}
& \int_{0}^{h(x)}\left|\partial_{x} b(x, y)\right|^{2} d y \leqslant C \int_{0}^{h(x)}\left|\partial_{x} \varphi(x, \xi)\right|^{2} d \xi, \\
& \left|\partial_{x} b\right|_{2}^{2}=\int_{0}^{L} \int_{0}^{h(x)}\left|\partial_{x} b(x, y)\right|^{2} d y d x \leqslant C\left|\partial_{x} \varphi\right|_{2}^{2}<\infty .
\end{aligned}
$$

It follows that $\partial_{x} b \in L^{2}(\Omega)$.

- On the other hand, $\partial_{z} b(x, z)=z / \eta(\varphi(x, z)) \in H^{1}(\Omega)$, since $\varphi \in$ $H^{1}(\Omega)$ and using $(9)$. 
$\triangleright$ Next we show that $b \in L^{\infty}(\Omega)$ : since $\partial_{z} b \in L^{2}(\Omega)$, we can write

$$
b(x, z)=b(x, 0)+\int_{0}^{z} \partial_{\xi} b(x, \xi) d \xi .
$$

By definition of $b$, we know that $b(x, 0)=0, \forall x \in[0, L]$. Therefore, the usual trace theorem for the Sobolev space $H^{1}(\Omega)$ implies that

$$
\begin{aligned}
|b(x, z)|^{2} & \leqslant z \int_{0}^{z}\left(\partial_{\xi} b(x, \xi)\right)^{2} d \xi \leqslant h_{M} \int_{0}^{h(x)}\left(\partial_{\xi} b(x, \xi)\right)^{2} d \xi=h_{M}\left|\partial_{z} b\right|_{L^{2}(0, h(x))}^{2} \\
& \leqslant C\left\|\partial_{z} b\right\|_{H^{1 / 2}(0, h(x))}^{2} \leqslant C\left\|\partial_{z} b\right\|_{1}^{2},
\end{aligned}
$$

thus

$$
|b|_{\infty}^{2} \leqslant C\left\|\partial_{z} b\right\|_{1}^{2}<\infty .
$$

It remains to prove the regularity of $\widetilde{a}, \widetilde{b}, \widetilde{c}, \widetilde{d}, \widetilde{e}$.

$\triangleright$ For the coefficients of the form $\widetilde{a}(x)=a(x, h(x)), \widetilde{b}(x)=b(x, h(x)), \widetilde{c}(x)=$ $c(x, h(x)), H^{1}$-regularity can be obtained using the same procedure as in the first part of the proof.

$\triangleright$ For $\widetilde{d}$ and $\widetilde{e}$, the key point of the proof is to observe that $H^{1}(0, L)$ (which is embedded in $\left.L^{\infty}(0, L)\right)$ is an algebra:

$$
(f, g) \in H^{1}(0, L)^{2} \Rightarrow f g \in H^{1}(0, L) .
$$

Recalling the definitions $\tilde{d}=\left(\widetilde{c}-\frac{\widetilde{b}^{2}}{\widetilde{a}}\right)$ and $\tilde{e}=\frac{\widetilde{b}}{\widetilde{a}}$, and using the fact that $\widetilde{a}, \widetilde{b}, \widetilde{c}$ belong to $H^{1}(0, L)$, we need to show that $1 / \widetilde{a}$ remains bounded. Since $\eta \leqslant \eta_{M}$, we have

$$
\widetilde{a}(x)=\int_{0}^{h(x)} \frac{1}{\eta(\varphi(x, \xi))} d \xi \geqslant \frac{h_{m}}{\eta_{M}} \quad \text { i.e. } \quad \frac{1}{\widetilde{a}} \leqslant C .
$$

From the regularity of $\widetilde{a}, \widetilde{b}, \widetilde{c}$, from the algebra structure and from (34), we deduce that

$$
\widetilde{d} \in H^{1}(0, L), \quad \tilde{e} \in H^{1}(0, L) .
$$

\subsection{Coercivity of the operator}

Lemma 4.2. Assume that the viscosity $\eta$ satisfies (9). Let $\widetilde{d}$ be defined by (21). It satisfies the following estimate:

$$
\forall x \in(0, L), \quad \tilde{d}(x) \geqslant \delta:=\frac{h_{m}^{3}}{12 \eta_{M}}>0 .
$$


Proof. By definition (21), $\widetilde{d}(x)$ can be written in the form:

$$
\widetilde{d}(x)=\widetilde{c}(x)-\frac{\widetilde{b}(x)^{2}}{\widetilde{a}(x)}=\int_{0}^{h(x)} \frac{z^{2}}{\eta(x, z)} d z-\frac{\left(\int_{0}^{h(x)} \frac{z}{\eta(x, z)} d z\right)^{2}}{\int_{0}^{h(x)} \frac{1}{\eta(x, z)} d z} .
$$

In order to prove the assertion, it suffices to prove that there exists $\delta>0$ such that

$$
\left(\int_{0}^{h} \frac{z^{2}}{\eta} d z\right)\left(\int_{0}^{h} \frac{1}{\eta} d z\right)-\left(\int_{0}^{h} \frac{z}{\eta} d z\right)^{2} \geqslant \delta\left(\int_{0}^{h} \frac{1}{\eta} d z\right) .
$$

Let us denote by $P$ the following polynomial

$$
\begin{aligned}
P: \nu \mapsto & \int_{0}^{h(x)}\left(\frac{z}{\sqrt{\eta(\varphi(x, z))}}+\frac{\nu}{\sqrt{\eta(\varphi(x, z))}}\right)^{2} d z \\
& =\int_{0}^{h(x)} \frac{z^{2}}{\eta(\varphi(x, z))}+\frac{\nu^{2}}{\eta(\varphi(x, z))}+\frac{2 z \nu}{\eta(\varphi(x, z))} d z .
\end{aligned}
$$

From (9), we get

$$
P(\nu) \geqslant \frac{1}{\eta_{M}} \int_{0}^{h(x)}\left(z^{2}+2 z \nu+\nu^{2}\right) d z=\frac{1}{3 \eta_{M}}\left(h(x)^{3}+3 h(x)^{2} \nu+3 h(x) \nu^{2}\right) .
$$

A simple study of the right-hand side polynomial proves that

$$
\forall \nu \in \mathbb{R}, \forall x \in(0, L), \quad h(x)^{2}+3 h(x) \nu+3 \nu^{2} \geqslant \frac{h(x)^{2}}{4},
$$

thus

$$
P(\nu) \geqslant \frac{h(x)^{3}}{12 \eta_{M}}, \quad \text { i.e. } \quad P(\nu)-\frac{h(x)^{3}}{12 \eta_{M}} \geqslant 0,
$$

therefore the discriminant of the polynomial

$$
P(\nu)-\frac{h(x)^{3}}{12 \eta_{M}}=\nu^{2} \int_{0}^{h} \frac{1}{\eta}+2 \nu \int_{0}^{h} \frac{z}{\eta}+\int_{0}^{h} \frac{z^{2}}{\eta}-\frac{h(x)^{3}}{12 \eta_{M}}
$$

is negative:

$4\left(\int_{0}^{h(x)} \frac{z d z}{\eta(\varphi(x, z))}\right)^{2}-4\left(\int_{0}^{h(x)} \frac{d z}{\eta(\varphi(x, z))}\right)\left[\left(\int_{0}^{h(x)} \frac{z^{2} d z}{\eta(\varphi(x, z))}\right)-\frac{h(x)^{3}}{12 \eta_{M}}\right] \leqslant 0$,

that is to say

$\left(\int_{0}^{h} \frac{z^{2}}{\eta} d z\right)\left(\int_{0}^{h} \frac{1}{\eta} d z\right)-\left(\int_{0}^{h} \frac{z}{\eta} d z\right)^{2} \geqslant \frac{h_{m}^{3}}{12 \eta_{M}}\left(\int_{0}^{h} \frac{1}{\eta} d z\right), \quad$ i.e. $\quad \tilde{d} \geqslant \frac{h_{m}^{3}}{12 \eta_{M}}>0$.

The two previous lemmas 4.1 (regularity of the coefficients) and 4.2 (coercivity of the operator) with formula (33) imply that $\partial_{x} p \in H^{1}(0, L)$, thus $p \in H^{2}(0, L)$. 


\subsection{Estimates of $|u|_{\infty}$ and $|v|_{2}$}

Lemma 4.3. Assume that the viscosity $\eta$ satisfies $(9)$. Assume $\varphi \in H^{1}(\Omega)$. The horizontal velocity $u$ given by (26b) satisfies

$$
|u|_{\infty} \leqslant C .
$$

Proof. The regularity of $u$ follows from the regularity of $p$, equation $26 \mathrm{~b}$ and the regularity of the coefficients (Lemma 4.1):

$$
u=\left(b-\frac{a \widetilde{b}}{\widetilde{a}}\right) \lambda \partial_{x} p+s\left(1-\frac{a}{\widetilde{a}}\right) \in X(\Omega) .
$$

Moreover, we know that $u$ is a combination of coefficients of the form $\int_{0}^{z} \xi / \eta(\varphi) d \xi$. Indeed

$$
|u|_{\infty} \leqslant\left(|b|_{\infty}+\frac{|a|_{\infty}|\widetilde{b}|_{\infty}}{\min _{x \in(0, L)} \widetilde{a}(x)}\right) \lambda\left|\partial_{x} p\right|_{\infty}+s\left(1+\frac{|a|_{\infty}}{\min _{x \in(0, L)} \widetilde{a}(x)}\right),
$$

and $\partial_{x} p$ is given by (33), thus:

$$
\lambda\left|\partial_{x} p\right|_{\infty} \leqslant \frac{1}{\min _{x \in(0, L)} \tilde{d}(x)}\left(s|\widetilde{e}|_{\infty}+\lambda|\widetilde{d}(0)||w|+s|\widetilde{e}(0)|\right) .
$$

Let us obtain estimates for these coefficients.

$\triangleright$ Using the boundedness hypothesis on $\eta$, and applying the Cauchy-Schwarz inequality and the fact that $\forall x \in(0, L), h(x) \leqslant h_{M}$, we can write for all $(x, z) \in \Omega$

$$
a(x, z)=\int_{0}^{z} \frac{d \xi}{\eta(\varphi(x, \xi))} \leqslant \frac{h_{M}}{\eta_{m}} \leqslant C, \quad \text { thus } \quad|a|_{\infty},|\widetilde{a}|_{\infty} \leqslant C .
$$

$\triangleright$ Similar computations for $b, c$ and $\widetilde{b}, \widetilde{c}$ give

$$
|b|_{\infty},|\widetilde{b}|_{\infty} \leqslant C, \quad|c|_{\infty},|\widetilde{c}|_{\infty} \leqslant C .
$$

$\triangleright$ Recalling definition (21) of $\widetilde{e}$, and using [34], it follows from (39):

$$
|\widetilde{e}|_{\infty}=\frac{|b|_{\infty}}{\min _{x \in(0, L)} \tilde{a}(x)} \leqslant C .
$$

$\triangleright$ We compute also from 29

$$
\lambda|w| \leqslant C .
$$


Thus, using all these estimates in (37), we get

$$
\lambda\left|\partial_{x} p\right|_{\infty} \leqslant C
$$

and combined with (36) and obvious estimates for $a, \widetilde{a}, b, \widetilde{b}$, we obtain the needed estimate:

$$
|u|_{\infty} \leqslant C
$$

Lemma 4.4. Assume that the viscosity $\eta$ satisfies (9). Assume $\varphi \in H^{1}(\Omega)$. Then the vertical velocity $v$ given by 26c) satisfies

$$
|v|_{2} \leqslant C\|\varphi\|_{1} \text {. }
$$

Proof. The regularity of $v$ follows from the regularity of $u$, equation 26c and the regularity of the coefficients (Lemma 4.1):

$$
v(x, z)=-\int_{0}^{z} \partial_{x} u(x, \xi) d \xi .
$$

From the Cauchy-Schwarz inequality, we deduce that

$$
|v|_{2} \leqslant h_{M}\left|\partial_{x} u\right|_{2}
$$

Let us introduce the coefficients $f=b-\frac{a \widetilde{b}}{\widetilde{a}}$ and $g=1-\frac{a}{\widetilde{a}}$, so that $u=\lambda f \partial_{x} p+s g$. Therefore

$$
\left|\partial_{x} u\right|_{2} \leqslant \lambda\left|\partial_{x} f\right|_{2}\left|\partial_{x} p\right|_{\infty}+\lambda|f|_{\infty}\left|\partial_{x}^{2} p\right|_{2}+s\left|\partial_{x} g\right|_{2},
$$

and $\partial_{x}^{2} p$ is given by taking the derivative of $(33)$ with respect to $x$ :

$$
\lambda\left|\partial_{x}^{2} p\right|_{2} \leqslant \frac{1}{\min _{x \in(0, L)} \widetilde{d}(x)}\left(s\left|\partial_{x} \widetilde{e}\right|_{2}+\lambda\left|\partial_{x} \widetilde{d}\right|_{2}\left|\partial_{x} p\right|_{\infty}\right) .
$$

Let us obtain estimates for each coefficient separately:

$\triangleright$ We have

$$
|f|_{\infty} \leqslant|\widetilde{b}|_{\infty}+C|a|_{\infty}|\widetilde{b}|_{\infty}
$$

$\triangleright$ It remains to obtain estimates of the derivatives of the coefficients with respect to $x$. We can compute $\partial_{x} a=\int_{0}^{y} \frac{\eta^{\prime}(\varphi)}{\eta(\varphi)^{2}} \partial_{x} \varphi$, and the CauchySchwarz inequality yields

$$
\left|\partial_{x} a\right|_{2}^{2} \leqslant \frac{\eta_{M}^{\prime 2}}{\eta_{m}^{4}} \int_{\Omega}\left(\int_{0}^{y} \partial_{x} \varphi(x, z) d z\right)^{2} \leqslant C \int_{\Omega} \int_{0}^{y}\left|\partial_{x} \varphi\right|^{2} \leqslant C\|\varphi\|_{1}^{2},
$$

and similar estimates for all the other coefficients:

$$
\begin{aligned}
\left|\partial_{x} a\right|_{2},\left|\partial_{x} \widetilde{a}\right|_{2} & \leqslant C\|\varphi\|_{1}, \\
\left|\partial_{x} b\right|_{2},\left|\partial_{x} \widetilde{b}\right|_{2} & \leqslant C\|\varphi\|_{1}, \\
\left|\partial_{x} c\right|_{2},\left|\partial_{x} \widetilde{c}\right|_{2} & \leqslant C\|\varphi\|_{1} .
\end{aligned}
$$


$\triangleright$ Let us write

$$
\partial_{x}\left(\frac{a}{\tilde{a}}\right)=\frac{\partial_{x} a \tilde{a}-a \partial_{x} \tilde{a}}{\widetilde{a}^{2}} .
$$

From (34), we know that $\tilde{a} \geqslant \frac{h_{m}}{\eta_{M}}$. This estimate combined with (38) and (48) suffices to prove that

$$
\left|\partial_{x}\left(\frac{a}{\tilde{a}}\right)\right|_{2} \leqslant C\|\varphi\|_{1}
$$

and

$$
\left|\partial_{x}\left(\frac{\widetilde{b}}{\widetilde{a}}\right)\right|_{2} \leqslant C\|\varphi\|_{1} .
$$

$\triangleright$ Since

$$
\begin{array}{ll}
\partial_{x} d=\partial_{x} c-\partial_{x} \widetilde{b} \frac{\tilde{b}}{\widetilde{a}}-\widetilde{b} \partial_{x}\left(\frac{\tilde{b}}{\bar{a}}\right), & \partial_{x} e=\partial_{x}\left(\frac{\tilde{b}}{\bar{a}}\right), \\
\partial_{x} f=\partial_{x} b-\partial_{x} a \frac{\widetilde{b}}{\overline{\widetilde{a}}}-a \partial_{x}\left(\frac{\widetilde{b}}{\overline{\widetilde{a}}}\right), & \partial_{x} g=\partial_{x}\left(\frac{a}{\overline{\widetilde{a}}}\right),
\end{array}
$$

it follows, using (48), 49, ,50) in 51, that

$$
\begin{array}{rlrl}
\left|\partial_{x} \widetilde{d}\right|_{2} & \leqslant C\|\varphi\|_{1}, & & \left|\partial_{x} \widetilde{e}\right|_{2} \leqslant C\|\varphi\|_{1}, \\
\left|\partial_{x} f\right|_{2} \leqslant C\|\varphi\|_{1}, & & \left|\partial_{x} g\right|_{2} \leqslant C\|\varphi\|_{1} .
\end{array}
$$

Putting (35), (52), (37) in (45) and (44), we deduce an estimate for each of the three terms in (44):

$\triangleright$ The first term is estimated by:

$$
\lambda\left|\partial_{x} f\right|_{2}\left|\partial_{x} p\right|_{\infty} \leqslant C\|\varphi\|_{1} .
$$

$\triangleright$ For the second term, we have:

$$
\frac{|f|_{\infty}}{\delta}\left(s\left|\partial_{x} \widetilde{e}\right|_{2}+\lambda\left|\partial_{x} \widetilde{d}\right|_{2}\left|\partial_{x} p\right|_{\infty}\right) \leqslant C\|\varphi\|_{1} .
$$

$\triangleright$ The third term $\left|\partial_{x} g\right|_{2}$ is exactly estimate $(52)$.

Therefore, using (43) and these three estimates for $\left|\partial_{x} u\right|_{2}$, we obtain:

$$
|v|_{2} \leqslant C\left|\partial_{x} u\right|_{2},
$$

which proves the lemma.

Remark 4.5. Observe that it is not straightforward to prove that $v \in L^{\infty}(\Omega)$ if $\varphi$ only lies in $H^{1}(\Omega)$. We get easily $|v|_{\infty} \leqslant C\left|\partial_{x} u\right|_{\infty}$, however the $H^{1}$-regularity of $\varphi$ is not sufficient to conclude.

Remark 4.6. Since (26a)-26b)-26c) are steady-state equations, the constants in the previous estimates are also independent of time, so that the $L^{\infty}(\Omega)$ and $L^{2}(\Omega)$-estimates of Lemma 4.3 and 4.4 can also be written in $L^{\infty}\left(0, T ; L^{\infty}(\Omega)\right)$ and $L^{\infty}\left(0, T ; L^{2}(\Omega)\right)$ for any $T>0$. 


\section{About the Cahn-Hilliard equation}

\subsection{Useful results and inequalities}

5.1.1. Boundary conditions and lift operator

We need a lift operator for the boundary value $\varphi_{l}$ of the order parameter $\varphi$.

Lemma 5.1. Let $\varphi_{l} \in H^{7 / 2}\left(\Gamma_{l}\right)$ satisfy Hypothesis 2.3. There exists $\hat{\varphi}_{l} \in$ $H^{7 / 2}(\Omega)$ such that the following conditions are satisfied

$$
\begin{aligned}
\left.\hat{\varphi}_{l}\right|_{\Gamma_{l}} & =\varphi_{l}, & \left.\nabla \hat{\varphi}_{l}\right|_{\Gamma_{0}} \cdot \boldsymbol{n} & =0, \\
\left.\frac{\alpha^{2}}{\lambda^{2}} \Delta \hat{\varphi}_{l}\right|_{\Gamma_{l}} & =F^{\prime}\left(\varphi_{l}\right), & \left.\nabla \Delta \hat{\varphi}_{l}\right|_{\Gamma_{0}} \cdot \boldsymbol{n} & =0 .
\end{aligned}
$$

Proof. For any $(x, z) \in \Omega$, let us define $\hat{\varphi}_{l}(x, z)=\chi(x) \varphi_{l}\left(\frac{h(0) z}{h(x)}\right)+\mathcal{F}$, where $\mathcal{F}$ is the solution of the following problem:

$$
\left\{\begin{array}{l}
\Delta \mathcal{F}=\frac{\lambda^{2}}{\alpha^{2}} \psi(x) F^{\prime}\left(\varphi_{l}\left(\frac{h(0) z}{h(x)}\right)\right)-\frac{h(0)}{h(x)} \chi(x) \varphi_{l}^{\prime \prime}\left(\frac{h(0) z}{h(x)}\right) \quad \text { in } \Omega, \\
\left.\mathcal{F}\right|_{\Gamma_{l}}=0 \\
\left.\nabla \mathcal{F}\right|_{\Gamma_{0}} \cdot \boldsymbol{n}=0
\end{array}\right.
$$

and the functions $\chi$ and $\psi$ are smooth functions satisfying the following conditions:

$$
\begin{aligned}
\chi(0) & =1, \quad \chi^{\prime}(0)=0, \quad \chi^{\prime \prime}(0)=0, \\
\psi(0) & =1, \\
\forall x \in[\tilde{\varepsilon}, L] \quad \chi(x) & =\chi^{\prime}(x)=\chi^{\prime \prime}(x)=\chi^{\prime \prime \prime}(x)=0, \\
\forall x \in[\tilde{\varepsilon}, L] \quad \psi(x) & =\psi^{\prime}(x)=0 .
\end{aligned}
$$

By regularity of the Laplacian [19, it follows immediately that $\mathcal{F} \in H^{7 / 2}(\Omega)$, thus $\hat{\varphi}_{l} \in H^{7 / 2}(\Omega)$.

Since $h^{\prime}(x) \equiv 0$ for $x \in[0, \tilde{\varepsilon}]$, the two last conditions imply that $\chi h^{\prime}$ is identically zero, and so are the other functions $\chi^{\prime} h^{\prime}, \chi^{\prime \prime} h^{\prime}, \chi^{\prime \prime \prime} h^{\prime}, \psi h^{\prime}, \psi^{\prime} h^{\prime}$ and $\chi h^{\prime \prime}$.

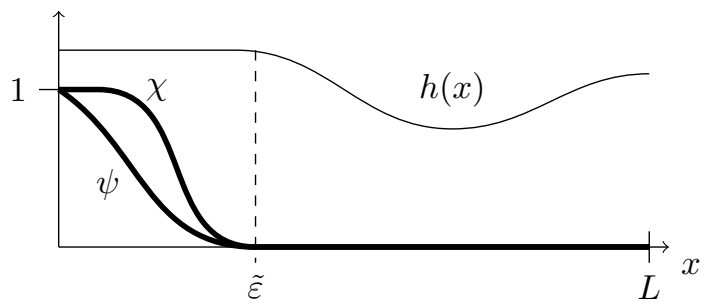

Figure 4: Possible shapes of function $\chi$ and $\psi$

Let us check that this function $\hat{\varphi}_{l}$ satisfies the claimed conditions:

- On $\Gamma_{l}, \mathcal{F}$ is zero, and since $\chi(0)=1, \hat{\varphi}_{l}$ has the right value. 
- On $\Gamma_{0}$, we know that $\left.\nabla \mathcal{F}\right|_{\Gamma_{0}} \cdot \boldsymbol{n}=0$, and we have to treat separately the three different boundaries for the other term:

- On $\Gamma_{b}, \partial_{y} \hat{\varphi}_{l}(x, 0)=\chi(x) \frac{h(0)}{h(x)} \varphi_{l}^{\prime}(0)=0$ by 19.

- On $\Gamma_{t}, h^{\prime}(x) \partial_{x} \hat{\varphi}_{l}(x, h(x))-\partial_{y} \hat{\varphi}_{l}(x, h(x))=h^{\prime}(x) \chi^{\prime}(x) \varphi_{l}(h(0))-$ $\chi(x) \frac{h^{\prime}(x)^{2} h(0)}{h(x)} \varphi_{l}^{\prime}(h(0))-\chi(x) \frac{h(0)}{h(x)} \varphi_{l}^{\prime}(h(0))=0$ by 19$)$ and using that $h^{\prime} \chi^{\prime}$ is identically zero.

- On $\Gamma_{r}$, all the terms of $\partial_{x} \hat{\varphi}_{l}(L, y)$ contain either $\chi(L)$ or $\chi^{\prime}(L)$, which are both equal to zero.

Let us compute the Laplacian of $\hat{\varphi}_{l}$. In order to improve the readibility, we denote $Y=\frac{h(0) y}{h(x)}$ :

$$
\begin{aligned}
\Delta \hat{\varphi}_{l}= & \chi^{\prime \prime}(x) \varphi_{l}(Y)-2 \frac{h^{\prime}(x) Y}{h(x)} \chi(x) \chi^{\prime}(x) \varphi_{l}^{\prime}(Y)+\frac{h^{\prime}(x)^{2} Y^{2}}{h(x)^{2}} \chi(x) \varphi_{l}^{\prime \prime}(Y) \\
& -\frac{h^{\prime \prime}(x) h^{2}(x)-2 h^{\prime}(x)^{2} h(x)}{h(x)^{3}} Y \chi^{\prime}(x) \varphi_{l}^{\prime}(Y)+\frac{h(0)}{h(x)} \chi(x) \varphi_{l}^{\prime \prime}(Y) \\
& +\frac{\lambda^{2}}{\alpha^{2}} \psi(x) F^{\prime}\left(\varphi_{l}(Y)\right)-\frac{h(0)}{h(x)} \chi(x) \varphi_{l}^{\prime \prime}(Y) .
\end{aligned}
$$

- We can compute the Laplacian on $\Gamma_{l}: \Delta \hat{\varphi}_{l}(0, y)=\frac{\lambda^{2}}{\alpha^{2}} F^{\prime}\left(\varphi_{l}(y)\right)$, since $\chi^{\prime}(0)=\chi^{\prime \prime}(0)=0, h^{\prime}(0)=0$ and $\psi(0)=1$.

- For $\Gamma_{0}$, we treat again each boundary separately:

- On $\Gamma_{b}$, we have to compute $\partial_{y} \Delta \hat{\varphi}_{l}$ at $(x, 0)$. Using that $y=0$ and $\varphi_{l}^{\prime}(0)=0$, we obtain that $\partial_{y} \Delta \hat{\varphi}_{l}(x, 0)=0$.

- On $\Gamma_{t}$, we compute $h^{\prime}(x) \partial_{x} \Delta \hat{\varphi}_{l}-\partial_{y} \Delta \hat{\varphi}_{l}$ at $(x, h(x))$. The terms in $h^{\prime}$ are multiplied by either $\chi, \chi^{\prime}, \chi^{\prime \prime}, \chi^{\prime \prime \prime}, \psi$, or $\psi^{\prime}$, and are therefore identically zero. For the other terms, we use the same arguments and that $\varphi_{l}^{\prime}(h(0))=0$ to conclude that the normal derivative of $\Delta \hat{\varphi}_{l}$ is zero on $\Gamma_{t}$.

- On $\Gamma_{r}$, we observe that $\chi(L)=\chi^{\prime}(L)=\chi^{\prime \prime}(L)=\chi^{\prime \prime \prime}(L)=\psi(L)=$ $\psi^{\prime}(L)=0$, thus $\partial_{x} \Delta \hat{\varphi}_{l}(L, y)=0$.

\subsubsection{Useful inequalities}

Sobolev embeddings. Let us recall the Poincaré inequality and usual Sobolev embeddings.

Proposition 5.2 (Poincaré inequality). Let $\Omega \subset \mathbb{R}^{2}$ defined by (2). For any $f \in H^{1}(\Omega)$ such that $\left.f\right|_{\Gamma_{i}}=0$ on one of the three parts $\Gamma_{l}, \Gamma_{b}, \Gamma_{r}$ of the boundary,

$$
|f|_{2} \leqslant C|\nabla f|_{2} \text {. }
$$


Proposition 5.3 (Sobolev embeddings). Let $\Omega \subset \mathbb{R}^{2}$ defined by (2). Then for any $2 \leqslant q<+\infty$, we have $H_{0}^{1}(\Omega) \hookrightarrow L^{q}(\Omega)$. More precisely, for any $f \in H^{1}(\Omega)$ with $\left.f\right|_{\Gamma_{i}}=0$ on one of the three parts $\Gamma_{l}, \Gamma_{b}, \Gamma_{r}$ of the boundary, we have

$$
|f|_{q} \leqslant C\|f\|_{1} .
$$

Equivalence of norms. (see [15] for a proof)

Proposition 5.4. Let $f \in H^{2}(\Omega)$ such that $\left.f\right|_{\Gamma_{i}}=0$ on one of the three parts $\Gamma_{l}$, $\Gamma_{b}, \Gamma_{r}$ of the boundary. We have

$$
\|f\|_{2} \leqslant C|\Delta f|_{2} .
$$

Trace estimates. (see [2] for a proof)

Proposition 5.5. For any $f \in H^{1}(\Omega)$ such that $\left.f\right|_{\Gamma_{i}}=0$ on one of the three parts $\Gamma_{l}, \Gamma_{b}, \Gamma_{r}$ of the boundary, we have

$$
|f|_{L^{2}\left(\Gamma_{l}\right)} \leqslant C|\nabla f|_{2} .
$$

Corollary 5.6. For $\varphi \in H^{2}(\Omega)$ satisfying the boundary conditions (18), we can apply this proposition to $\partial_{x} \varphi$, since $\left.\partial_{x} \varphi\right|_{\Gamma_{r}}=0$, and deduce that

$$
\left|\partial_{x} \varphi\right|_{L^{2}\left(\Gamma_{l}\right)} \leqslant C\left|\nabla \partial_{x} \varphi\right|_{2}
$$

and if we combine this relation with Proposition 5.4, we obtain

$$
\left|\partial_{x} \varphi\right|_{L^{2}\left(\Gamma_{l}\right)} \leqslant C|\Delta \varphi|_{2}
$$

\subsection{Galerkin approximations}

Let us build Galerkin approximations of $\varphi$ and $\mu$. Since $H^{1}(\Omega)$ is a separable Hilbert space, there exists an Hilbertian basis $\left(\psi_{i}\right)_{i \geqslant 1}$ of $H^{1}(\Omega)$. The functions $\psi_{i}$ can be chosen to be eigenfunctions of the Laplacian $-\Delta$ with the boundary conditions

$$
\left.\frac{\partial \psi_{i}}{\partial \boldsymbol{n}}\right|_{\Gamma_{0}}=0,\left.\quad \psi_{i}\right|_{\Gamma_{l}}=0,
$$

and we denote by $\lambda_{i}$ the corresponding eigenvalues. As far as the regularity of the functions $\psi_{i}$ is concerned, we have $\psi_{i} \in H^{2}(\Omega)$ (this result can be deduced from [15]). We define $\Psi_{n}=\operatorname{Span}\left(\psi_{1}, \cdots, \psi_{n}\right)$, and $\mathbb{P}_{\Psi_{n}}$ the orthogonal projector on $\Psi_{n}$ in $L^{2}(\Omega)$. As a projector, $\mathbb{P}_{\Psi_{n}}$ satisfies:

$$
\left(\mathbb{P}_{\Psi_{n}} f, g\right)=\left(f, \mathbb{P}_{\Psi_{n}} g\right), \quad \forall(f, g) \in L^{2}(\Omega)^{2},
$$

where $(\cdot, \cdot)$ denotes the scalar product in $L^{2}(\Omega)$.

Recalling that $\hat{\varphi}_{l} \in H^{7 / 2}(\Omega)$ is a lifting of the boundary condition $\varphi_{l}$ defined in Lemma 5.1. we consider the following approximation of $\varphi$ :

$$
\varphi_{n}(t)=\sum_{i=1}^{n} \beta_{i}(t) \psi_{i}+\hat{\varphi}_{l}
$$

where $\beta_{i}$ are unknown functions to be determined. In this setting, $\varphi_{n}(0)-\hat{\varphi}_{l}$ is the orthogonal projection of $\varphi_{0}-\hat{\varphi}_{l}$ on $\Psi_{n}$. Let us introduce the following auxiliary function $a$, which will be useful in order to define $\mu_{n}$ : 
Proposition 5.7. There exists $a \in H^{1}(\Omega)$ such that

$$
\left.a\right|_{\Gamma_{l}}=F^{\prime}\left(\varphi_{l}\right),\left.\quad \nabla a \cdot n\right|_{\Gamma_{0}}=0 .
$$

Proof. Let us define $a$ by $a(x, z)=F^{\prime}\left(\varphi_{l}\left(\frac{h(0) z}{h(x)}\right)\right)$. We check that $a$ satisfies the claimed conditions.

- On $\Gamma_{l}, a(0, z)=F^{\prime}\left(\varphi_{l}(z)\right)$.

- On $\Gamma_{b}, \partial_{z} a(x, 0)=-\frac{h(0)}{h(x)} \varphi_{l}^{\prime}(0) F^{\prime \prime}\left(\varphi_{l}(0)\right)=0$ by 19$)$.

- On $\Gamma_{t}$, the normal derivative is written $h^{\prime}(x) \partial_{x} a(x, h(x))-\partial_{z} a(x, h(x))$. The two terms are again equal to zero thanks to 19 .

- On $\Gamma_{r}, \partial_{x} a(L, z)=-\frac{h^{\prime}(L) h(0) z}{h(L)^{2}} \varphi_{l}^{\prime}\left(\frac{h(0) z}{h(x)}\right) F^{\prime \prime}\left(\varphi_{l}\left(\frac{h(0) z}{h(x)}\right)\right)$, which is also zero since $h^{\prime}(L)=0$.

Taking (30)-(31) into account, let us define $\left(\varphi_{n}, \mu_{n}\right)$ as the solution of the following weak problem:

Problem 5.8. Find $\varphi_{n}=\sum_{i=1}^{n} \beta_{i}(t) \psi_{i}+\hat{\varphi}_{l}$ and $\mu_{n}$ such that

$$
\begin{gathered}
\lambda \int_{\Omega} \partial_{t} \varphi_{n} \psi+\int_{\Omega} \frac{1}{\lambda \mathcal{P} e} \mathcal{B}\left(\varphi_{n}\right) \nabla \mu_{n} \nabla \psi+\int_{\Omega} \boldsymbol{u}_{\varphi_{n}} \cdot \nabla \varphi_{n} \psi=0, \quad \forall \psi \in \Psi_{n}, \\
\mu_{n}=-\frac{\alpha^{2}}{\lambda^{2}} \Delta \varphi_{n}+a+\mathbb{P}_{\Psi_{n}}\left(F^{\prime}\left(\varphi_{n}\right)-a\right),
\end{gathered}
$$

with the initial condition $\left.\varphi_{n}\right|_{t=0}=\varphi_{0}$ and the boundary conditions

$$
\left.\mu_{n}\right|_{\Gamma_{l}}=0,\left.\quad \varphi_{n}\right|_{\Gamma_{l}}=\varphi_{l},\left.\quad \nabla \mu_{n} \cdot \boldsymbol{n}\right|_{\Gamma_{0}}=\left.\nabla \varphi_{n} \cdot \boldsymbol{n}\right|_{\Gamma_{0}}=0,
$$

and where $\boldsymbol{u}_{\varphi_{n}}$ is defined for each $\varphi_{n}$ by the formulas 222-23) and 25.

This problem can indeed be obtained from 26) because the boundary term vanishes, as proved in the following proposition.

Proposition 5.9. Let $\left(\varphi_{n}, \mu_{n}\right)$ solution of Problem 5.8. Then the boundary term coming from the integration by parts cancels:

$$
\int_{\Gamma} B\left(\varphi_{n}\right) \nabla \mu_{n} \cdot \boldsymbol{n} \psi=0 .
$$


Proof. $\triangleright$ On $\Gamma_{0}$, we can compute $\left.\nabla \mu_{n} \cdot \boldsymbol{n}\right|_{\Gamma_{0}}$, using that the functions $\psi_{i}$ are eigenfunctions of $-\Delta$ :

$$
\begin{aligned}
& \left.\nabla \mu_{n} \cdot \boldsymbol{n}\right|_{\Gamma_{0}}=-\left.\frac{\alpha^{2}}{\lambda^{2}} \nabla \Delta \varphi_{n} \cdot \boldsymbol{n}\right|_{\Gamma_{0}}+\underbrace{\left.\nabla a \cdot \boldsymbol{n}\right|_{\Gamma_{0}}}_{=0 \text { by Def. } 5.7}+\underbrace{\left.\nabla \mathbb{P}_{\Psi_{n}}\left(F^{\prime}\left(\varphi_{n}\right)-a\right) \cdot \boldsymbol{n}\right|_{\Gamma_{0}}}_{=0, \text { since } \mathbb{P}_{\Psi_{n}}\left(F^{\prime}\left(\varphi_{n}\right)-a\right) \in \Psi_{n}} \\
& =-\left.\frac{\alpha^{2}}{\lambda^{2}} \nabla\left(\sum_{i=1}^{n} \beta_{i} \lambda_{i} \psi_{i}\right) \cdot \boldsymbol{n}\right|_{\Gamma_{0}}+\underbrace{\left.\nabla \Delta \hat{\varphi}_{l} \cdot \boldsymbol{n}\right|_{\Gamma_{0}}}_{=0 \text { by Lem. 5.1 }} .
\end{aligned}
$$

Since $\psi_{i} \in \Psi_{n}$ for any $i \leqslant n$, we have $\left.\nabla \psi_{i} \cdot \boldsymbol{n}\right|_{\Gamma_{0}}=0$, we deduce $\left.\nabla \mu_{n} \cdot \boldsymbol{n}\right|_{\Gamma_{0}}=$ 0 .

$\triangleright$ On $\Gamma_{l}$, the boundary term is also equal to zero, since $\psi \in \Psi_{n}$, and thus vanishes on $\Gamma_{l}$.

Observe that the weak formulation (58)-(59) is well-defined since $\psi_{i} \in H_{0}^{1}(\Omega)$ implies that $\mu_{n} \in H^{1}(\Omega)$. Indeed, the functions $\psi_{i}$ are eigenfunctions of $-\Delta$, thus the regularity follows from definition 59 .

Remark 5.10. Observe that the chosen approximation (59) of $\mu$ satisfies the same boundary conditions as $\mu$, because of the definition of $\hat{\varphi}_{l}$ in Lemma 5.1. Moreover, if it converges, it is towards $\mu=-\frac{\alpha^{2}}{\lambda^{2}} \Delta \varphi+F^{\prime}(\varphi)$, since $\mathbb{P}_{\Psi_{n}}$ converges towards the identity. Indeed, $F^{\prime}\left(\varphi_{n}\right)-a$ satisfies the right boundary conditions in $\Phi_{0}^{1}$ (by construction of a, see Proposition 5.7):

$$
\begin{aligned}
& \triangleright F^{\prime}\left(\varphi_{n}\right)-a=0 \text { on } \Gamma_{l}, \\
& \triangleright \nabla\left(F^{\prime}\left(\varphi_{n}\right)-a\right) \cdot \boldsymbol{n}=0 \text { on } \Gamma_{0} .
\end{aligned}
$$

Lemma 5.11. For $n \in \mathbb{N}$, there exist $t_{n}>0$ and $\left(\beta_{i}\right)_{1 \leqslant i \leqslant n} \in \mathcal{C}^{1}\left(0, t_{n}\right)$ such that $\varphi_{n}(t)=\sum_{i=1}^{n} \beta_{i}(t) \psi_{i}+\hat{\varphi}_{l}$ is a solution of Problem 5.8 .

Proof. Replacing $\varphi_{n}$ by its expression as a function of $\beta_{i}$, the system (58)-(59) becomes:

$$
\begin{aligned}
& \lambda \sum_{i=1}^{n} \beta_{i}^{\prime}(t) \int_{\Omega} \psi_{i} \psi+ \int_{\Omega} \frac{1}{\lambda \mathcal{P} e} \mathcal{B}\left(\sum_{i=1}^{n} \beta_{i}(t) \psi_{i}+\hat{\varphi}_{l}\right) \nabla \mu_{n} \nabla \psi \\
&+\sum_{i=1}^{n} \beta_{i}(t) \int_{\Omega} \boldsymbol{u}_{\left\{\sum_{i=1}^{n} \beta_{i}(t) \psi_{i}+\hat{\varphi}_{l}\right\}} \cdot \nabla \psi_{i} \psi=0, \quad \forall \psi \in \Psi_{n} \\
& \mu_{n}=-\frac{\alpha^{2}}{\lambda^{2}} \sum_{i=1}^{n} \beta_{i}(t) \lambda_{i} \psi_{i}+a+\mathbb{P}_{\Psi_{n}} F^{\prime}\left(\sum_{i=1}^{n} \beta_{i}(t) \psi_{i}+\hat{\varphi}_{l}-a\right) .
\end{aligned}
$$

This formulation is an ordinary differential equation on $\left(\beta_{i}\right)_{1 \leqslant i \leqslant n}$. The functions $\mathcal{B}$ and $F^{\prime}$ are of class $\mathcal{C}^{1}$ on $\mathbb{R}$. Moreover, the function $\boldsymbol{u}$ as a function of $\varphi_{n}$ given by 26b-26c)-26a is also $\mathcal{C}^{1}$ on $\mathbb{R}_{+}$(with respect to time): indeed, $u_{\varphi_{n}}$ 
is given as a combination of coefficients of the form $\int_{0}^{z} \xi / \eta\left(\varphi_{n}(x, \xi)\right) d \xi$, and the function $\eta$ is $\mathcal{C}^{1}$ by assumption (9). The second component of the velocity $v$ is given as a function of $u$, and is also $\mathcal{C}^{1}$ on $\mathbb{R}_{+}$. Therefore, the Cauchy-Lipschitz theorem ensures the existence of a unique solution $\left(\beta_{i}\right)_{1 \leqslant i \leqslant n}$ on a time interval $\left[0, t_{n}\right)$.

Last, let us introduce another auxiliary function $b$, which is another lifting of the boundary condition $\varphi_{l}$ and will be used to apply Poincaré inequality:

Proposition 5.12. There exists $b \in H^{2}(\Omega)$ such that for some small $\varepsilon>0$ that will be determined later,

$$
\left.b\right|_{\Gamma_{l}}=\varphi_{l},\left.\quad \nabla b \cdot \boldsymbol{n}\right|_{\Gamma_{0}}=0, \quad\left|\partial_{x} b\right|_{2}<\varepsilon .
$$

Proof. Let us define $b$ by $b(x, z)=\varphi_{l}\left(\frac{h(0) z}{h(x)}\right)$. Let us check that $b$ satisfies the claimed conditions. The first ones are the sames as in Proposition 5.7, and are satisfied in the same way:

- On $\Gamma_{l}, b(0, z)=\varphi_{l}(z)$.

- On $\Gamma_{b}, \partial_{z} b(x, 0)=\frac{h(0)}{h(x)} \varphi_{l}^{\prime}(0)=0$ by $(19)$.

- On $\Gamma_{t}$, the normal derivative is written $h^{\prime}(x) \partial_{x} b(x, h(x))-\partial_{z} b(x, h(x))$. The two terms are again equal to zero thanks to 19 .

- On $\Gamma_{r}, \partial_{x} b(L, z)=-\frac{h^{\prime}(L) h(0) z}{h(L)^{2}} \varphi_{l}^{\prime}\left(\frac{h(0) z}{h(x)}\right)$, which is also zero since $h^{\prime}(L)=$ 0 .

Last, we observe that

$$
\left|\partial_{x} b\right|_{2}^{2}=\int_{\Omega} \frac{h^{\prime}(x)^{2} h(0)^{2} z^{2}}{h(x)^{4}}\left|\varphi_{l}^{\prime}\left(\frac{h(0) z}{h(x)}\right)\right|^{2} \mathrm{~d} x \mathrm{~d} z \leqslant C\left|\varphi_{l}^{\prime}\right|_{L^{2}(0,1)}^{2},
$$

and thus by (3) and (19), this term can be arbitrarily small. Therefore, in order to ensure the smallness of $\left|\partial_{x} b\right|_{2}$, we have to choose $\varepsilon$ sufficiently small. Therefore, this determines the smallness assumption on $\bar{\varepsilon}$ in 19 in Hypothesis 2.3 .

\subsection{Equation on $\varphi$}

Let us now focus on obtaining estimates of $\varphi_{n}, \mu_{n}$ in appropriate function spaces. In the sequel, we drop the subscripts ${ }_{n}$ for the sake of readability, and we write $\varphi, \mu$ instead of $\varphi_{n}, \mu_{n}$.

Lemma 5.13. For $\varphi$ and $\mu$ solutions of (58)-60), the following applies:

$$
\begin{aligned}
\lambda \frac{d}{d t}\left(\frac{\alpha^{2}}{2 \lambda^{2}}|\nabla \varphi|_{2}^{2}+\int_{\Omega} F(\varphi)\right) & +\left(\frac{\mathcal{B}_{m}}{\lambda \mathcal{P} e}-1\right)|\nabla \mu|_{2}^{2} \\
& \leqslant C\left(\left(|u|_{\infty}^{2}+|v|_{2}^{2}\right)|\Delta \varphi|_{2}^{2}+|v|_{2}^{2}\|b\|_{2}^{2}\right) .
\end{aligned}
$$


Proof. Let us take $\psi=\mu \in \Psi_{n}$ in the weak formulation (58). Using Definition (59) for $\mu$, we get

$$
\lambda \underbrace{\int_{\Omega} \partial_{t} \varphi\left(-\frac{\alpha^{2}}{\lambda^{2}} \Delta \varphi+a+\mathbb{P}_{\Psi_{n}}\left(F^{\prime}(\varphi)-a\right)\right)}_{=: A}+\underbrace{\frac{1}{\lambda \mathcal{P} e} \int_{\Omega} \mathcal{B}(\varphi)|\nabla \mu|^{2}}_{=: B}=\underbrace{-\int_{\Omega} \boldsymbol{u} \cdot \nabla \varphi \mu}_{=: D} .
$$

Let us obtain estimates for each term $A, B, D$ :

$\triangleright$ The $A$-term is composed of two parts:

$$
A=\underbrace{-\frac{\alpha^{2}}{\lambda^{2}} \int_{\Omega} \partial_{t} \varphi \Delta \varphi+\int_{\Omega} \partial_{t} \varphi}_{=: A_{1}}+\underbrace{\left.\int_{\Omega} \partial_{t} \varphi \mathbb{P}_{\Psi_{n}}\left(F^{\prime}(\varphi)-a\right)\right)}_{=: A_{2}} .
$$

$\star$ For $A_{1}$, we use integration by parts:

$$
\begin{aligned}
A_{1} & =-\frac{\alpha^{2}}{\lambda^{2}} \int_{\Omega} \partial_{t} \varphi \Delta \varphi+\int_{\Omega} \partial_{t} \varphi a \\
& =\frac{\alpha^{2}}{2 \lambda^{2}} \frac{d}{d t}|\nabla \varphi|_{2}^{2}-\frac{\alpha^{2}}{\lambda^{2}} \int_{\Gamma} \partial_{t} \varphi \nabla \varphi \cdot \boldsymbol{n}+\int_{\Omega} \partial_{t} \varphi a .
\end{aligned}
$$

The boundary condition $\left.\nabla \psi_{i} \cdot \boldsymbol{n}\right|_{\Gamma_{0}}=0$, and the fact that $\varphi_{l}$ is independent of $t$ allow us to treat the boundary term:

$$
-\frac{\alpha^{2}}{\lambda^{2}} \int_{\Gamma} \underbrace{\partial_{t} \varphi}_{=0 \text { on } \Gamma_{l}} \underbrace{\nabla \varphi \cdot \boldsymbol{n}}_{=0 \text { on } \Gamma_{0}}=0
$$

thus

$$
A_{1}=\frac{\alpha^{2}}{2 \lambda^{2}} \frac{d}{d t}|\nabla \varphi|_{2}^{2}+\int_{\Omega} \partial_{t} \varphi a .
$$

$\star$ For the second term, observe that from the time-independency of $\hat{\varphi}_{l}$ and $\psi_{i} \in \Psi_{n}$, it yields

$$
\mathbb{P}_{\Psi_{n}} \partial_{t} \varphi=\mathbb{P}_{\Psi_{n}}\left(\sum_{i=1}^{n} \beta_{i}^{\prime}(t) \psi_{i}\right)=\sum_{i=1}^{n} \beta_{i}^{\prime}(t) \psi_{i}=\partial_{t} \varphi
$$

Now, we use property (57) and 64:

$A_{2}=\left(\partial_{t} \varphi, \mathbb{P}_{\Psi_{n}}\left(F^{\prime}(\varphi)-a\right)\right)=\left(\mathbb{P}_{\Psi_{n}} \partial_{t} \varphi, F^{\prime}(\varphi)-a\right)=\left(\partial_{t} \varphi, F^{\prime}(\varphi)-a\right)$.

Thus, $A_{2}$ can be expressed as a time derivative plus a second term which will cancel with the last term in 63):

$$
A_{2}=\int_{\Omega} \partial_{t} \varphi F^{\prime}(\varphi)-\int_{\Omega} \partial_{t} \varphi a=\frac{d}{d t} \int_{\Omega} F(\varphi)-\int_{\Omega} \partial_{t} \varphi a .
$$


$\triangleright$ The $B$-term is trivially estimated using that $\mathcal{B}(\varphi) \geqslant \mathcal{B}_{m}$ (see (17)):

$$
B=\frac{1}{\lambda \mathcal{P} e} \int_{\Omega} \mathcal{B}(\varphi)|\nabla \mu|^{2} \geqslant \frac{\mathcal{B}_{m}}{\lambda \mathcal{P} e}|\nabla \mu|_{2}^{2} .
$$

$\triangleright$ For the $D$-term, we split it into two terms:

$$
D=\underbrace{\int_{\Omega} u \partial_{x} \varphi \mu}_{=D_{1}}+\underbrace{\int_{\Omega} v \partial_{y} \varphi \mu}_{=D_{2}} .
$$

* We use Poincaré inequality (53) and Young's inequality

$$
\begin{aligned}
D_{1} & =\int_{\Omega} u \partial_{x} \varphi \mu \leqslant|u|_{\infty}\left|\partial_{x} \varphi\right|_{2}|\mu|_{2} \leqslant C|u|_{\infty}\left|\partial_{x} \varphi\right|_{2}|\nabla \mu|_{2} \\
& \leqslant \frac{1}{2}|\nabla \mu|_{2}^{2}+C|u|_{\infty}^{2}\left|\partial_{x} \varphi\right|_{2}^{2} .
\end{aligned}
$$

Now, observe that $\partial_{x} \varphi$ is zero on $\Gamma_{r}$, and thus the Poincaré inequality yields

$$
\left|\partial_{x} \varphi\right|_{2}^{2} \leqslant L\left|\partial_{x}^{2} \varphi\right|_{2}^{2} \leqslant C|\Delta \varphi|_{2}^{2}
$$

Combining these two estimates, we obtain

$$
D_{1} \leqslant \frac{1}{2}|\nabla \mu|_{2}^{2}+C|u|_{\infty}^{2}|\Delta \varphi|_{2}^{2}
$$

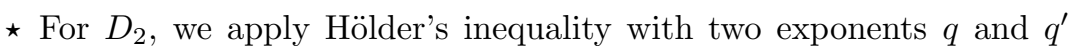
strictly greater than 2 such that $\frac{1}{q}+\frac{1}{q^{\prime}}=\frac{1}{2}$ and the Sobolev inequality 54 for $|\mu|_{q^{\prime}}$ with the Poincaré inequality (53):

$D_{2}=\int_{\Omega} v \partial_{y} \varphi \mu \leqslant|v|_{2}\left|\partial_{y} \varphi\right|_{q}|\mu|_{q^{\prime}} \leqslant C|v|_{2}\left|\partial_{y} \varphi\right|_{q}\|\mu\|_{1} \leqslant C|v|_{2}\left|\partial_{y} \varphi\right|_{q}|\nabla \mu|_{2}$.

Now, we use (54) for $\left|\partial_{y} \varphi\right|_{q}$, and Young's inequality

$$
D_{2} \leqslant C|v|_{2}\left\|\partial_{y} \varphi\right\|_{1}|\nabla \mu|_{2} \leqslant \frac{1}{2}|\nabla \mu|_{2}^{2}+C|v|_{2}^{2}\left\|\partial_{y} \varphi\right\|_{1}^{2} .
$$

It remains to apply (55) with a function equals to zero on $\Gamma_{l}$. This is done using $b$ defined in Definition 5.12 Since $\left\|\partial_{y}(\varphi-b)\right\|_{1} \leqslant$ $C|\Delta(\varphi-b)|_{2}$, we have

$$
D_{2} \leqslant \frac{1}{2}|\nabla \mu|_{2}^{2}+C|v|_{2}^{2}\left(|\Delta \varphi|_{2}^{2}+\|||\|_{2}^{2}\right) .
$$

Putting 63, 65), 66), 67), 68 into 62 , and rearranging terms, we get

$$
\begin{aligned}
\lambda \frac{d}{d t}\left(\frac{\alpha^{2}}{2 \lambda^{2}}|\nabla \varphi|_{2}^{2}+\int_{\Omega} F(\varphi)\right) & +\left(\frac{\mathcal{B}_{m}}{\lambda \mathcal{P} e}-1\right)|\nabla \mu|_{2}^{2} \\
& \leqslant C\left(\left(|u|_{\infty}^{2}+|v|_{2}^{2}\right)|\Delta \varphi|_{2}^{2}+|v|_{2}^{2}\|b\|_{2}^{2}\right) .
\end{aligned}
$$

This proves inequality 61. 


\subsection{Equation on $\mu$}

Lemma 5.14. For $\varphi$ and $\mu$ solutions of (58)-(60), the following inequality applies:

$$
\frac{\alpha^{2}}{\lambda^{2}}|\nabla \varphi|_{2}^{2}+F_{3}(0) \int_{\Omega} F(\varphi) \leqslant \frac{C}{\lambda^{2}}|\Delta \varphi|_{2}^{2}+\frac{1}{2}|\nabla \mu|_{2}^{2}+C|\nabla \varphi|_{2}^{2 r}+C|\nabla \varphi|_{2}^{2}+T_{l} .
$$

where $T_{l}$ is independent of $\varphi, \mu$ and of time $t$, and is given by:

$$
T_{l}=C\left(1+\|b\|_{1}^{2}+\left|\hat{\varphi}_{l}\right|_{2}^{2}+\|b\|_{1}^{2 r}\right)+|a|_{2}\left|\hat{\varphi}_{l}\right|_{2}+C\left(\frac{1}{\lambda^{2}}+1\right)\|b\|_{1}^{2} .
$$

Proof. If we multiply 59 by $\varphi$, we get

$$
\underbrace{(\mu, \varphi)}_{=: A}=\underbrace{\left(-\frac{\alpha^{2}}{\lambda^{2}} \Delta \varphi+a, \varphi\right)}_{=: B}+\underbrace{\left(\mathbb{P}_{\Psi_{n}}\left(F^{\prime}(\varphi)-a\right), \varphi\right)}_{=: D} \text {. }
$$

As before, let us treat each term separately.

$\triangleright$ For $B$, we use integration by parts, and obtain:

$$
B=\frac{\alpha^{2}}{\lambda^{2}}|\nabla \varphi|_{2}^{2}-\underbrace{\frac{\alpha^{2}}{\lambda^{2}} \int_{\Gamma} \varphi \nabla \varphi \cdot \boldsymbol{n}}_{=: B_{1}}+\underbrace{\int_{\Omega} a \varphi}_{(\star)} .
$$

Observe that since $\left.\nabla \varphi \cdot \boldsymbol{n}\right|_{\Gamma_{0}}=0$, the boundary term $B_{1}$ is zero on $\Gamma \backslash \Gamma_{l}$. Using (56) and Young's inequality, it follows:

$$
\begin{aligned}
\left|B_{1}\right| & =\frac{\alpha^{2}}{\lambda^{2}}\left|\int_{\Gamma_{l}} \varphi_{l} \partial_{x} \varphi\right| \leqslant \frac{\alpha^{2}}{\lambda^{2}}\left|\varphi_{l}\right|_{L^{2}\left(\Gamma_{l}\right)}\left|\partial_{x} \varphi\right|_{L^{2}\left(\Gamma_{l}\right)} \\
& \leqslant \frac{C}{\lambda^{2}}\left|\varphi_{l}\right|_{L^{2}\left(\Gamma_{l}\right)}|\Delta \varphi|_{2} \leqslant \frac{C}{\lambda^{2}}\left(|\Delta \varphi|_{2}^{2}+\|b\|_{1}^{2}\right)
\end{aligned}
$$

where we used $b$ as a lift of $\varphi_{l}$.

$\triangleright$ For the $D$-term, let us use the projector property 57 and the fact that $\varphi-\hat{\varphi}_{l} \in \Psi_{n}$ (i.e. $\mathbb{P}_{\Psi_{n}}\left(\varphi-\hat{\varphi}_{l}\right)=\varphi-\hat{\varphi}_{l}$, and thus $\left.\mathbb{P}_{\Psi_{n}} \varphi=\varphi-\left(\operatorname{Id}-\mathbb{P}_{\Psi_{n}}\right) \hat{\varphi}_{l}\right)$ :

$$
\begin{aligned}
D & =\left(\mathbb{P}_{\Psi_{n}}\left(F^{\prime}(\varphi)-a\right), \varphi\right)=\left(F^{\prime}(\varphi)-a, \mathbb{P}_{\Psi_{n}} \varphi\right) \\
& =\underbrace{\left(F^{\prime}(\varphi), \varphi\right)}_{=: D_{1}} \underbrace{-(a, \varphi)}_{-(\star)} \underbrace{-\left(F^{\prime}(\varphi),\left(\operatorname{Id}-\mathbb{P}_{\Psi_{n}}\right) \hat{\varphi}_{l}\right)}_{=: D_{2}} \underbrace{+\left(a,\left(\operatorname{Id}-\mathbb{P}_{\Psi_{n}}\right) \hat{\varphi}_{l}\right)}_{=: D_{3}} .
\end{aligned}
$$

The term $-(\star)$ cancels with the one in 72 . Hypothesis 16 with $\gamma=0$ yields

$$
D_{1}=\int_{\Omega} F^{\prime}(\varphi) \varphi \geqslant \int_{\Omega} F_{3}(0) F(\varphi)-F_{4}(0)|\Omega| \geqslant \int_{\Omega} F_{3}(0) F(\varphi)-C .
$$


As far as $D_{2}$ is concerned, we use the fact that $\operatorname{Id}-\mathbb{P}_{\Psi_{n}}$ is a projector, thus its operator norm (in $L^{2}(\Omega)$ ) is equal to 1 . We also use the property (15) for $\left|F^{\prime}(\varphi)\right|$ and (54) for $|\varphi|_{2 r}^{r}$ to obtain (if $r>1$ ):

$$
\begin{aligned}
\left|D_{2}\right| & =\left|\left(F^{\prime}(\varphi),\left(\operatorname{Id}-\mathbb{P}_{\Psi_{n}}\right) \hat{\varphi}_{l}\right)\right| \leqslant\left|\hat{\varphi}_{l}\right|_{2}\left|F^{\prime}(\varphi)\right|_{2} \\
& \leqslant C\left|\hat{\varphi}_{l}\right|_{2}\left(F_{1}|\varphi|_{2 r}^{r}+F_{2}|\Omega|\right) \leqslant C\left|\hat{\varphi}_{l}\right|_{2}\left(\|\varphi\|_{1}^{r}+1\right) \\
& \leqslant C\left|\hat{\varphi}_{l}\right|_{2}\left(|\nabla \varphi|_{2}^{r}+\|b\|_{1}^{r}+1\right) \\
& \leqslant C\left(\left|\hat{\varphi}_{l}\right|_{2}^{2}+|\nabla \varphi|_{2}^{2 r}+\|b\|_{1}^{2 r}+1\right),
\end{aligned}
$$

where in the third line, we used the lifting $b$ of the boundary condition $\varphi_{l}$ defined in Definition 5.12 to apply Poincaré inequality. Observe that we proved the following estimate on $F^{\prime}(\varphi)$, which will be used in the following:

$$
\left|F^{\prime}(\varphi)\right|_{2}^{2} \leqslant C\left(|\nabla \varphi|_{2}^{2 r}+\|b\|_{1}^{2 r}+1\right) .
$$

Last, we use again the fact that the operator norm of $\mathrm{Id}-\mathbb{P}_{\Psi_{n}}$ is equal to 1 , and write

$$
D_{3} \leqslant|a|_{2}\left|\hat{\varphi}_{l}\right|_{2} .
$$

$\triangleright$ For the $A$-term, Young's inequality combined with the Poincaré inequality for $\varphi$ (using $b$ as a lifting of $\varphi_{l}$ ) and (53) for $\mu$ yields:

$$
\begin{aligned}
A & =\int_{\Omega} \mu \varphi \leqslant|\mu|_{2}|\varphi|_{2} \leqslant C|\nabla \mu|_{2}\left(C|\nabla \varphi|_{2}+\|b\|_{1}\right) \\
& \leqslant C|\nabla \mu|_{2}\left(|\nabla \varphi|_{2}+\|b\|_{1}\right) \leqslant \frac{1}{2}|\nabla \mu|_{2}^{2}+C\left(|\nabla \varphi|_{2}^{2}+\|b\|_{1}^{2}\right) .
\end{aligned}
$$

Putting (72)-(78) in 71), and rearranging terms, it follows:

$$
\begin{aligned}
\frac{\alpha^{2}}{\lambda^{2}}|\nabla \varphi|_{2}^{2} & +F_{3}(0) \int_{\Omega} F(\varphi) \leqslant \frac{C}{\lambda^{2}}|\Delta \varphi|_{2}^{2}+\frac{1}{2}|\nabla \mu|_{2}^{2}+C|\nabla \varphi|_{2}^{2 r}+C|\nabla \varphi|_{2}^{2} \\
& +C\left(\left|\varphi_{l}\right|_{L^{2}\left(\Gamma_{l}\right)}^{2}+\left|\hat{\varphi}_{l}\right|_{2}^{2}+\|b\|_{1}^{2 r}\right)+|a|_{2}\left|\hat{\varphi}_{l}\right|_{2}+C\left(1+\frac{1}{\lambda^{2}}\right)\|b\|_{1}^{2}+C,
\end{aligned}
$$

which is the inequality 70 we claimed.

Lemma 5.15. For $\varphi$ and $\mu$ solutions of (58)-(60), the following estimate applies for any $\theta, \kappa>0$ :

$$
\left(\frac{\alpha^{2}}{\lambda^{2}}-3 \kappa\right)|\Delta \varphi|_{2}^{2} \leqslant \frac{1}{2}|\nabla \mu|_{2}^{2}+\frac{1}{2}|\nabla \varphi|_{2}^{2}+\frac{C}{\kappa}|\nabla \varphi|_{2}^{2 r}+S_{l},
$$

where $S_{l}$ is independent of $\varphi, \mu$ and of time $t$, and is given by:

$$
S_{l}=\frac{C}{\kappa}\left(\|b\|_{1}^{2 r}+1\right)+\frac{1}{\kappa}|a|_{2}^{2} .
$$


Proof. Multiplying (59) by $-\Delta \varphi$ and integrating by parts, we get

$$
\frac{\alpha^{2}}{\lambda^{2}}|\Delta \varphi|_{2}^{2}=\underbrace{-(\mu, \Delta \varphi)}_{=: A}+\underbrace{\int_{\Omega} \mathbb{P}_{\Psi_{n}}\left(F^{\prime}(\varphi)-a\right) \Delta \varphi}_{=: B}+\underbrace{(a, \Delta \varphi)}_{=: D} .
$$

$\triangleright$ We treat the $D$-term with Young's inequality with some constant $\kappa>0$ :

$$
D=(a, \Delta \varphi) \leqslant \frac{1}{\kappa}|a|_{2}^{2}+\kappa|\Delta \varphi|_{2}^{2} .
$$

$\triangleright$ For the $B$-term, we use the projector property (57) and Young's inequality to obtain the following estimate:

$$
\begin{aligned}
B=\left(\mathbb{P}_{\Psi_{n}}\left(F^{\prime}(\varphi)-a\right), \Delta \varphi\right) & \leqslant\left. F^{\prime}(\varphi)\right|_{2}|\Delta \varphi|_{2}+|a|_{2}|\Delta \varphi|_{2} \\
& \leqslant \frac{1}{\kappa}|a|_{2}^{2}+\kappa|\Delta \varphi|_{2}^{2}+\kappa|\Delta \varphi|_{2}^{2}+\frac{C}{\kappa}\left|F^{\prime}(\varphi)\right|_{2}^{2} .
\end{aligned}
$$

Then we can use $(76)$ to deduce that

$$
B \leqslant 2 \kappa|\Delta \varphi|_{2}^{2}+\frac{1}{\kappa}|a|_{2}^{2}+\frac{C}{\kappa}\left(|\nabla \varphi|_{2}^{2 r}+\|b\|_{1}^{2 r}+1\right) .
$$

$\triangleright$ As far as the $A$-term is concerned, it is computed by integration by parts:

$$
A=-(\mu, \Delta \varphi)=\underbrace{\int_{\Omega} \nabla \mu \nabla \varphi}_{=: A_{1}}-\int_{\Gamma} \underbrace{\mu}_{=0 \text { on } \Gamma_{l}} \underbrace{\nabla \varphi \cdot \boldsymbol{n}}_{=0 \text { on } \Gamma_{0}} .
$$

Thanks to Young's inequality, we have

$$
A=-(\nabla \mu, \nabla \varphi) \leqslant \frac{1}{2}|\nabla \mu|_{2}^{2}+\frac{1}{2}|\nabla \varphi|_{2}^{2} .
$$

Finally, we use $817-83)$ in 80$)$ to obtain

$$
\left(\frac{\alpha^{2}}{\lambda^{2}}-3 \kappa\right)|\Delta \varphi|_{2}^{2} \leqslant \frac{1}{2}|\nabla \mu|_{2}^{2}+\frac{1}{2}|\nabla \varphi|_{2}^{2}+\frac{C}{\kappa}|\nabla \varphi|_{2}^{2 r}+\frac{C}{\kappa}\left(\|b\|_{1}^{2 r}+1\right)+\frac{1}{\kappa}|a|_{2}^{2} .
$$

This concludes the proof.

\subsection{Convergence results}

\subsubsection{A priori estimates}

Let us sum (61), (70) and $c_{3} \times(79)$, where $c_{3}$ is a positive constant that will be determined in the sequel. We obtain

$$
\begin{gathered}
\lambda \frac{d}{d t}\left(\frac{\alpha^{2}}{2 \lambda^{2}}|\nabla \varphi|_{2}^{2}+\int_{\Omega} F(\varphi)\right)+\left(\frac{\mathcal{B}_{m}}{\lambda \mathcal{P} e}-\frac{3}{2}-\frac{c_{3}}{2}\right)|\nabla \mu|_{2}^{2}+\left(\frac{\alpha^{2}}{\lambda^{2}}-C-\frac{c_{3}}{2}\right)|\nabla \varphi|_{2}^{2} \\
+\left(c_{3}\left(\frac{\alpha^{2}}{\lambda^{2}}-3 \kappa\right)-\frac{C}{\lambda^{2}}\right)|\Delta \varphi|_{2}^{2}+F_{3}(0) \int_{\Omega} F(\varphi) \\
\leqslant C\left(\left(|u|_{\infty}^{2}+|v|_{2}^{2}\right)|\Delta \varphi|_{2}^{2}+|v|_{2}^{2}\|b \mid\|_{2}^{2}\right)+\left(C+\frac{c_{3} C}{\kappa}\right)|\nabla \varphi|_{2}^{2 r}+c_{3} S_{l}+T_{l} .
\end{gathered}
$$


To control the right hand side member of 84 we recall that we proved in 32 that

$$
|u|_{\infty} \leqslant C, \quad|v|_{2} \leqslant C\|\varphi\|_{1} .
$$

We apply the Poincaré inequality choosing $b$ as a lift for $\varphi$ to gain

$$
|u|_{\infty}^{2} \leqslant C, \quad|v|_{2}^{2} \leqslant C|\nabla \varphi|_{2}^{2}+C\|b\|_{1}^{2} .
$$

Estimate (84) becomes

$$
\begin{aligned}
& \lambda \frac{d}{d t}\left(\frac{\alpha^{2}}{2 \lambda^{2}}|\nabla \varphi|_{2}^{2}+\int_{\Omega} F(\varphi)\right)+\left(\frac{\mathcal{B}_{m}}{\lambda \mathcal{P} e}-\frac{3}{2}-\frac{c_{3}}{2}\right)|\nabla \mu|_{2}^{2}+\left(\frac{\alpha^{2}}{\lambda^{2}}-C-\frac{c_{3}}{2}\right)|\nabla \varphi|_{2}^{2} \\
& +\left(c_{3}\left(\frac{\alpha^{2}}{\lambda^{2}}-3 \kappa\right)-\frac{C}{\lambda^{2}}-C\right)|\Delta \varphi|_{2}^{2}+F_{3}(0) \int_{\Omega} F(\varphi) \\
& \quad \leqslant C|\nabla \varphi|_{2}^{2}|\Delta \varphi|_{2}^{2}+C\left(1+\frac{c_{3}}{\kappa}\right)|\nabla \varphi|_{2}^{2 r}+C+\frac{C}{\lambda^{2}}+\frac{C c_{3}}{\kappa}
\end{aligned}
$$

In order to ensure

$$
\begin{aligned}
& \frac{\mathcal{B}_{m}}{\lambda \mathcal{P} e}-\frac{3}{2}-\frac{c_{3}}{2} \geqslant \frac{\mathcal{B}_{m}}{2 \lambda \mathcal{P} e}, \\
& \frac{\alpha^{2}}{\lambda^{2}}-C-\frac{c_{3}}{2} \geqslant \frac{\alpha^{2}}{2 \lambda^{2}}, \\
& c_{3}\left(\frac{\alpha^{2}}{\lambda^{2}}-3 \kappa\right)-\frac{C}{\lambda^{2}}-C \geqslant \frac{c_{3} \alpha^{2}}{2 \lambda^{2}},
\end{aligned}
$$

we will choose $c_{3}, \lambda$ such that

$$
\begin{aligned}
& \frac{3}{2}+\frac{c_{3}}{2} \leqslant \frac{\mathcal{B}_{m}}{2 \lambda \mathcal{P} e}, \\
& C+\frac{c_{3}}{2} \leqslant \frac{\alpha^{2}}{2 \lambda^{2}}, \\
& 3 \kappa c_{3}+\frac{C}{\lambda^{2}}+C \leqslant \frac{c_{3} \alpha^{2}}{2 \lambda^{2}} .
\end{aligned}
$$

We choose $c_{3}$ with $c_{3} \alpha^{2}$ large enough such that the third condition can be rewritten as

$$
3 \kappa c_{3}+C \leqslant \underbrace{\left(\frac{c_{3} \alpha^{2}}{2}-C\right)}_{>0} \frac{1}{\lambda^{2}} .
$$

Newt, choosing $\lambda>0$ small enough ensures the required inequalities.

Estimate (86) becomes

$$
\begin{aligned}
& \lambda \frac{d}{d t}\left(\frac{\alpha^{2}}{2 \lambda^{2}}|\nabla \varphi|_{2}^{2}+\int_{\Omega} F(\varphi)\right)+\frac{\mathcal{B}_{m}}{2 \lambda \mathcal{P} e}|\nabla \mu|_{2}^{2}+\frac{\alpha^{2}}{2 \lambda^{2}}|\nabla \varphi|_{2}^{2}+\frac{c_{3} \alpha^{2}}{2 \lambda^{2}}|\Delta \varphi|_{2}^{2}+F_{3}(0) \int_{\Omega} F(\varphi) \\
& \quad \leqslant C|\nabla \varphi|_{2}^{2}|\Delta \varphi|_{2}^{2}+C\left(1+\frac{c_{3}}{\kappa}\right)|\nabla \varphi|_{2}^{2 r}+C+\frac{C}{\lambda^{2}}+\frac{C c_{3}}{\kappa}
\end{aligned}
$$


Let us define for all $t \geqslant 0$,

$$
\begin{aligned}
& \mathcal{Y}(t)=\frac{\alpha^{2}}{2 \lambda^{2}}|\nabla \varphi(t)|_{2}^{2}+\int_{\Omega} F(\varphi(t)), \\
& \mathcal{Z}(t)=\frac{\alpha^{2}}{2 \lambda^{2}}|\nabla \varphi(t)|_{2}^{2}+|\nabla \mu(t)|_{2}^{2}+|\Delta \varphi(t)|_{2}^{2}+\int_{\Omega} F(\varphi(t)),
\end{aligned}
$$

so that $0 \leqslant \mathcal{Y}(t) \leqslant \mathcal{Z}(t)$, since $F>0$ (by assumption 13 ).

Lemma 5.16. There exists strictly positive constants $C_{1}, C_{2}$ and $f: \mathbb{R} \rightarrow \mathbb{R}$ an increasing continuous function satisfying $f(0)=0$ satisfying

- $C_{1}>0$;

- there exists $M>0$ such that

$\star f(M)<C_{1} / 2$;

$\star C_{2}<M C_{1} / 2$.

such that the a priori estimate (87) can be rewritten in the following form:

$$
\mathcal{Y}^{\prime}(t)+C_{1} \mathcal{Z}(t) \leqslant f(\mathcal{Y}(t)) \mathcal{Z}(t)+C_{2}
$$

Proof. In order to rewrite (87) as the inequality (88), we have to set apart the linear terms (with respect to $\mathcal{Z}$ ) and the nonlinear terms (which will appear in $f(\mathcal{Y}) \mathcal{Z})$.

Defining

$$
C_{1}:=\frac{1}{\lambda} \min \left\{\frac{\mathcal{B}_{m}}{2 \lambda \mathcal{P} e}, 1, \frac{c_{3} \alpha^{2}}{2 \lambda^{2}}, F_{3}(0)\right\}>0
$$

and

$$
C_{2}:=\frac{C}{\lambda}\left(1+\frac{1}{\lambda^{2}}+\frac{c_{3}}{\kappa}\right)>0,
$$

we rewrite 87 as

$$
\mathcal{Y}^{\prime}(t)+C_{1} \mathcal{Z}(t) \leqslant f(\mathcal{Y}(t)) \mathcal{Z}(t)+C_{2}
$$

We can also give explicitely the form of $f$, which is given, up to a multiplicative constant, by

$$
f(x)=C \frac{2 \lambda^{2}}{\alpha^{2}} x+C\left(1+\frac{c_{3}}{\kappa}\right)\left(\frac{2 \lambda^{2}}{\alpha^{2}}\right)^{2 r} x^{r-1} .
$$

For $r>1$, it is always possible to find $M>0$ such that $f(M)<C_{1} / 2$.

It remains to impose that the right-hand side is controlled by $C_{1}$, i.e. that $C_{2}<M C_{1} / 2$. This is achieved by imposing some smallness conditions on $\lambda$. Indeed, if $\lambda \sim 0$ then we have $C_{1} \sim \frac{1+F_{3}(0)}{\lambda}$ and $C_{2} \sim \frac{C}{\lambda}\left(1+\frac{c_{3}}{\kappa}\right)$. It is then possible to find $M>0$ satisfying the desired property, since the two constants are of the same order in $\lambda$. This concludes the proof.

From now on, let us come back to the notations with the subscripts ${ }_{n}$ introduced in Section 5.2, denoting the Galerkin approximations. The proof of the main theorem consists in showing that $t_{n}=+\infty$ for any $n \geqslant 1$, and that $\varphi_{n}$ converges in appropriate function spaces. 
Lemma 5.17. For any $n \in \mathbb{N}$, under a smallness assumption on $\lambda$ and Hypothesis 2.3, there exists $C>0$ such that for any $T>0$,

$$
\left\|\varphi_{n}\right\|_{L^{\infty}\left(\mathbb{R}^{+} ; H^{1}(\Omega)\right)} \leqslant C, \quad\left\|\varphi_{n}\right\|_{L^{2}\left(0, T ; H^{2}(\Omega)\right)} \leqslant C T, \quad\left\|\mu_{n}\right\|_{L^{2}\left(0, T ; \Phi_{0}^{1}\right)} \leqslant C T .
$$

Proof. Let $n \in \mathbb{N}, T>0$. The assumptions are enough to apply Lemma 5.16 with Proposition 7.1 (given in Appendix) which implies that $\mathcal{Y}_{n} \in L^{\infty}(0, T)$ with a bound independent of $T$, and $\mathcal{Z}_{n} \in L^{1}(0, T)$ with a bound depending on $T$. From this, we deduce several results on $\varphi_{n}, \mu_{n}$ :

- The quantity $\nabla \varphi_{n}$ is bounded in $L^{\infty}\left(0, \infty ; L^{2}(\Omega)\right)$, uniformly with respect to $n$.

- The quantities $\nabla \mu_{n}, \nabla \varphi_{n}$ and $\Delta \varphi_{n}$ are bounded in $L_{\mathrm{loc}}^{2}\left(0, \infty ; L^{2}(\Omega)\right)$, uniformly with respect to $n$.

- Furthermore, applying the Poincaré inequality to $\varphi_{n}$ allows us to control the whole $H^{1}(\Omega)$-norm by the $L^{2}$-norm of the gradient.

- As far as the $H^{2}$-norm of $\varphi_{n}$ is concerned, we know by Proposition 5.4 that it is equivalent to the $L^{2}$-norm of the Laplacian, and thus controlling $\left|\Delta \varphi_{n}\right|_{2}$ is enough to control the whole $H^{2}(\Omega)$-norm.

- For $\mu_{n}$, the Poincaré inequality (53) also allows us to control the $H^{1}$-norm by the $L^{2}$-norm of the gradient.

From these arguments, we conclude that there exists $C>0$ such that for any $T>0$, estimate $(89)$ is satisfied.

Let us observe that the first estimate of 89 is enough to show that the time interval $\left(0, t_{n}\right)$ on which the functions $\varphi_{n}$ exist is $(0,+\infty)$.

Estimates (89) are not enough to conclude for the convergence of the nonlinear terms and of the initial condition $\varphi_{n}(0)$. Therefore, some more regularity on $\varphi_{n}$ and $\partial_{t} \varphi_{n}$ will be proved in the next subsection. We also note that the value of the scaling $\lambda$ is now fixed: the constants $C$ which appear from now can depend on $\lambda$.

\subsection{2. $H^{3}$-estimate for $\varphi$}

Lemma 5.18. For any $n \in \mathbb{N}$, under a smallness assumption on $\lambda$, there exists $C>0$ such that for any $T>0$

$$
\left\|\varphi_{n}\right\|_{L^{2}\left(0, T ; H^{3}(\Omega)\right)} \leqslant C T+C .
$$

Proof. We compute the gradient of (59):

$$
\frac{\alpha^{2}}{\lambda^{2}} \nabla \Delta \varphi_{n}-\nabla a=\underbrace{\nabla \mathbb{P}_{\Psi_{n}}\left(F^{\prime}\left(\varphi_{n}\right)-a\right)}_{=: A}-\nabla \mu_{n} .
$$


$\triangleright$ Let us prove that $|A|_{2}^{2} \leqslant\left|\nabla F^{\prime}\left(\varphi_{n}\right)\right|_{2}^{2}$. The difficulty here is to switch the two operators $\nabla \cdot$ and $\mathbb{P}_{\Psi_{n}} \cdot$. We have by integration by parts

$$
\begin{aligned}
&|A|_{2}^{2}= \int_{\Omega} \nabla \mathbb{P}_{\Psi_{n}}\left(F^{\prime}\left(\varphi_{n}\right)-a\right) \cdot \nabla \mathbb{P}_{\Psi_{n}}\left(F^{\prime}\left(\varphi_{n}\right)-a\right) \\
&=-\int_{\Omega} \Delta \mathbb{P}_{\Psi_{n}}\left(F^{\prime}\left(\varphi_{n}\right)-a\right) \mathbb{P}_{\Psi_{n}}\left(F^{\prime}\left(\varphi_{n}\right)-a\right) \\
& \quad+\int_{\Gamma} \nabla \mathbb{P}_{\Psi_{n}}\left(F^{\prime}\left(\varphi_{n}\right)-a\right) \cdot \boldsymbol{n} \mathbb{P}_{\Psi_{n}}\left(F^{\prime}\left(\varphi_{n}\right)-a\right),
\end{aligned}
$$

where the boundary term on $\Gamma$ cancels since $\mathbb{P}_{\Psi_{n}}\left(F^{\prime}\left(\varphi_{n}\right)-a\right) \in \Psi_{n}$. Let us denote $\Phi^{1} \ni F^{\prime}\left(\varphi_{n}\right)-a=\sum_{i=1}^{\infty} \gamma_{i} \psi_{i}$. We have $\mathbb{P}_{\Psi_{n}}\left(F^{\prime}\left(\varphi_{n}\right)-a\right)=\sum_{i=1}^{n} \gamma_{i} \psi_{i}$. Thus, we can compute

$$
|A|_{2}^{2}=-\int_{\Omega} \sum_{i=1}^{n} \lambda_{i} \gamma_{i} \psi_{i} \sum_{i=1}^{n} \gamma_{i} \psi_{i}
$$

and since the $\psi_{i}$ are orthogonal, we have

$$
\begin{aligned}
|A|_{2}^{2} & =-\sum_{i=1}^{n}\left(\lambda_{i} \gamma_{i} \psi_{i}, \gamma_{i} \psi_{i}\right)=-\sum_{i=1}^{n}\left(\Delta \gamma_{i} \psi_{i}, \gamma_{i} \psi_{i}\right)=\sum_{i=1}^{n}\left(\nabla \gamma_{i} \psi_{i}, \nabla \gamma_{i} \psi_{i}\right) \\
& =\left(\mathbb{P}_{\Psi_{n}} \nabla\left(F^{\prime}\left(\varphi_{n}\right)-a\right), \mathbb{P}_{\Psi_{n}} \nabla\left(F^{\prime}\left(\varphi_{n}\right)-a\right)\right) \\
& =\left|\mathbb{P}_{\Psi_{n}} \nabla\left(F^{\prime}\left(\varphi_{n}\right)-a\right)\right|_{2}^{2} \leqslant\left|\nabla\left(F^{\prime}\left(\varphi_{n}\right)-a\right)\right|_{2}^{2} \leqslant\left|\nabla F^{\prime}\left(\varphi_{n}\right)\right|_{2}^{2}+|\nabla a|_{2}^{2},
\end{aligned}
$$

since the operator norm of $\mathbb{P}_{\Psi_{n}}$ is equal to 1 .

$\triangleright$ It follows from hypothesis 15 on $F$ that:

$$
\left|\nabla F^{\prime}\left(\varphi_{n}\right)\right|_{2}^{2} \leqslant \int_{\Omega}\left(F_{1}\left|\varphi_{n}\right|^{r-1}+F_{2}\right)^{2}\left|\nabla \varphi_{n}\right|^{2} \leqslant C\left(\left|\nabla \varphi_{n}\right|_{2}^{2}+\left|\varphi_{n}^{r-1} \nabla \varphi_{n}\right|_{2}^{2}\right) .
$$

Since $r>1$, the Hölder inequality implies

$$
\begin{aligned}
\left|\nabla F^{\prime}\left(\varphi_{n}\right)\right|_{2}^{2} & \leqslant C\left(\left|\nabla \varphi_{n}\right|_{2}^{2}+\left(\int_{\Omega}\left|\varphi_{n}^{2(r-1)}\right|^{q}\right)^{1 / q}\left(\int_{\Omega}\left|\nabla \varphi_{n}\right|^{2 q^{\prime}}\right)^{1 / q^{\prime}}\right) \\
& =C\left(\left|\nabla \varphi_{n}\right|_{2}^{2}+\left|\varphi_{n}\right|_{2(r-1) q}^{2(r-1)}\left|\nabla \varphi_{n}\right|_{2 q^{\prime}}^{2}\right),
\end{aligned}
$$

with $\frac{1}{q}+\frac{1}{q^{\prime}}=1$, for any $q>1$. Let $q=\frac{1}{r-1}$. Then $2(r-1) q \geqslant 2$, thus $H^{1}(\Omega) \hookrightarrow L^{2(r-1) q}(\Omega)$ and $2 q^{\prime} \geqslant 2$, thus $H^{1}(\Omega) \hookrightarrow L^{2 q^{\prime}}(\Omega)$. We finally obtain

$$
|A|_{2}^{2} \leqslant C\left(\left|\nabla \varphi_{n}\right|_{2}^{2}+\left\|\varphi_{n}\right\|_{1}^{r-1}\left\|\varphi_{n}\right\|_{2}^{2}\right)+\alpha^{2}\left|\nabla \Delta \hat{\varphi}_{l}\right|_{2}^{2}\left|\nabla F^{\prime}\left(\varphi_{n}\right)\right|_{2}^{2}+|\nabla a|_{2}^{2},
$$


$\triangleright$ At last, taking the $L^{2}$-norm of $(91$, it follows from $(92)$ that

$$
\frac{\alpha^{2}}{\lambda^{2}}\left|\nabla \Delta \varphi_{n}\right|_{2}^{2} \leqslant C\left(\left|\nabla \mu_{n}\right|_{2}^{2}+\left|\nabla \varphi_{n}\right|_{2}^{2}+\left\|\varphi_{n}\right\|_{1}^{r-1}\left\|\varphi_{n}\right\|_{2}^{2}\right)+|\nabla a|_{2}^{2},
$$

This estimate combined with 89 and the regularity of $\hat{\varphi}_{l}$ (Lemma 5.1) allows us to conclude that estimate 90 is satisfied.

5.5.3. Time derivative estimate for $\varphi$

Lemma 5.19. For any $n \in \mathbb{N}$, under a smallness assumption on $\lambda$, there exists $C>0$ such that for any $T>0$,

$$
\left\|\frac{d \varphi_{n}}{d t}\right\|_{L^{2}\left(0, T ; H^{-1}(\Omega)\right)} \leqslant C T+C .
$$

Proof. We introduce the dual operator $\mathbb{P}_{\Psi_{n}}^{*}$ of $\mathbb{P}_{\Psi_{n}}$. Equation (58) can be rewritten in the following form:

$\left(\lambda \partial_{t} \varphi_{n}, \mathbb{P}_{\Psi_{n}} \chi\right)+\left(\boldsymbol{u}_{\varphi_{n}} \cdot \nabla \varphi_{n}, \mathbb{P}_{\Psi_{n}} \chi\right)-\frac{1}{\lambda \mathcal{P} e}\left(\operatorname{div}\left(\mathcal{B}\left(\varphi_{n}\right) \nabla \mu_{n}\right), \mathbb{P}_{\Psi_{n}} \chi\right)=0, \quad \forall \chi \in \Phi_{0}^{1}$,

which becomes

$$
\lambda \frac{d \varphi_{n}}{d t}=-\mathbb{P}_{\Psi_{n}}^{*}\left(u_{\varphi_{n}} \partial_{x} \varphi_{n}+v_{\varphi_{n}} \partial_{z} \varphi_{n}-\frac{1}{\lambda \mathcal{P} e} \operatorname{div}\left(\mathcal{B}\left(\varphi_{n}\right) \nabla \mu_{n}\right)\right) .
$$

Let us treat each term separately:

$\triangleright$ By Proposition 3.4 we have

$$
u_{\varphi_{n}} \in L^{\infty}\left(0, T ; H^{1}(\Omega)\right), \quad v_{\varphi_{n}} \in L^{\infty}\left(0, T ; L^{2}(\Omega)\right) .
$$

Moreover, previous estimate 90 implies that $\varphi_{n}$ belongs to $L^{2}\left(0, T ; H^{3}(\Omega)\right)$. By a classical result on the multiplicative algebra structure of the Sobolev spaces proved e.g. in [21], we deduce that

$$
u_{\varphi_{n}} \partial_{x} \varphi_{n} \in L^{2}\left(0, T ; H^{1}(\Omega)\right), \quad v_{\varphi_{n}} \partial_{z} \varphi_{n} \in L^{2}\left(0, T ; L^{2}(\Omega)\right),
$$

with the following estimate:

$$
\begin{aligned}
& \left\|u_{\varphi_{n}} \partial_{x} \varphi_{n}\right\|_{L^{2}\left(0, T ; H^{1}\right)}+\left\|v_{\varphi_{n}} \partial_{z} \varphi_{n}\right\|_{L^{2}\left(0, T ; L^{2}\right)} \\
& \quad \leqslant C\left(\left\|u_{\varphi_{n}}\right\|_{L^{\infty}\left(0, T ; H^{1}\right)}+\left\|v_{\varphi_{n}}\right\|_{L^{2}\left(0, T ; L^{2}\right)}+\left\|\varphi_{n}\right\|_{L^{2}\left(0, T ; H^{3}\right)}\right) .
\end{aligned}
$$

$\triangleright$ Furthermore, since $\mathcal{B} \leqslant \mathcal{B}_{m}$ :

$$
\left\|\operatorname{div}\left(\mathcal{B}\left(\varphi_{n}\right) \nabla \mu_{n}\right)\right\|_{H^{-1}} \leqslant \mathcal{B}_{m}\left|\nabla \mu_{n}\right|_{2} .
$$

It follows the claimed estimate 93 . 


\subsubsection{Final convergence results}

It is now possible to prove the main theorem 3.3 , re-stated here for the sake of readibility:

Theorem. Let $T>0, \varphi_{l}$ satisfying Hypothesis $2.3, \varphi_{0} \in H^{3}(\Omega)$ satisfying 18), F satisfy the assumptions stated in Section 2.2. Under a smallness assumption on $\lambda$, there exists a solution $(p, \boldsymbol{u}, \varphi, \mu)$ of Problem 3.2.

Proof. From the previous Lemmas 5.17, 5.18 and 5.19 (i.e. estimates 890 , (90), (93)), we obtain the following convergence results (up to a subsequence):

$$
\begin{array}{cl}
\varphi_{n} \rightarrow \varphi & \text { in } L^{\infty}\left(\mathbb{R}^{+} ; H^{1}(\Omega)\right) \quad \text { *-weak, } \\
\varphi_{n} \rightarrow \varphi & \text { in } L_{\mathrm{loc}}^{2}\left(\mathbb{R}^{+} ; H^{3}(\Omega)\right) \quad \text { weak, } \\
\mu_{n} \rightarrow \mu & \text { in } L_{\mathrm{loc}}^{2}\left(\mathbb{R}^{+} ; \Phi_{0}^{1}\right) \quad \text { weak, } \\
\frac{d \varphi_{n}}{d t}-\frac{d \varphi}{d t} & \text { in } L_{\mathrm{loc}}^{2}\left(\mathbb{R}^{+} ; H^{-1}(\Omega)\right) \quad \text { weak. }
\end{array}
$$

Moreover, Proposition 3.4 combined with the previous global convergence result on $\varphi$ implies the following convergence results (up to a subsequence):

$$
\begin{array}{lll}
u_{n} \rightarrow u & \text { in } L^{\infty}\left(\mathbb{R}^{+} ; \mathcal{X}(\Omega)\right) & \text { *-weak, } \\
v_{n} \rightarrow v & \text { in } L^{\infty}\left(\mathbb{R}^{+} ; L^{2}(\Omega)\right) & \text { *-weak, } \\
p_{n} \rightarrow p & \text { in } L^{\infty}\left(\mathbb{R}^{+} ; H^{2}(0, L)\right) & \text { *-weak. }
\end{array}
$$

Therefore, from the convergences of $\varphi_{n}$, we deduce

$$
\varphi_{n} \rightarrow \varphi \quad \text { in } L_{\text {loc }}^{2}\left(\mathbb{R}^{+} ; H^{2}(\Omega)\right) \quad \text { strong. }
$$

Furthermore, by a classical embedding result due to [25], we deduce from (90) and 93 that for any $T>0$

$$
\begin{array}{lll}
\varphi_{n} \rightarrow \varphi & \text { in } \mathcal{C}^{0}\left([0, T) ; L^{2}(\Omega)\right) & \text { strong, } \\
\varphi_{n} \rightarrow \varphi & \text { in } \mathcal{C}^{0}\left([0, T) ; H^{1}(\Omega)\right) & \text { weak. }
\end{array}
$$

Therefore, we can conclude for the convergence of the nonlinear terms:

- Since $\varphi_{n}$ converges strongly in $\mathcal{C}^{0}\left([0, T) ; L^{2}(\Omega)\right) \cap L_{\text {loc }}^{2}\left(\mathbb{R}^{+} ; H^{2}(\Omega)\right)$, the nonlinear terms $B\left(\varphi_{n}\right)$ and $F^{\prime}\left(\varphi_{n}\right)$ converge strongly in $\mathcal{C}^{0}\left([0, T) ; L^{2}(\Omega)\right)$.

- As far as the convection term $\boldsymbol{u}_{\varphi_{n}} \cdot \nabla \varphi_{n}$ is concerned, we know from Lemmas 4.3 and 4.4 that $\boldsymbol{u}_{\varphi_{n}}$ is bounded in $L^{\infty}\left(\mathbb{R}^{+} ; L^{2}(\Omega)\right)$. From the strong convergence of $\nabla \varphi_{n}$ in $L_{\text {loc }}^{2}\left(\mathbb{R}^{+} ; L^{2}(\Omega)\right)$, we conclude the convergence of $\boldsymbol{u}_{\varphi_{n}} \cdot \nabla \varphi_{n}$.

Lastly, we deduce from the last convergence result that $\varphi_{n}(0)$ converges weakly to $\varphi(0)$ in $H^{1}(\Omega)$, and thus $\varphi(0)=\varphi_{0}$ because $\mathbb{P}_{\Psi_{n}}$ converges to the identity for the strong topology of operators. For the boundary conditions on $\varphi$, the previous 
convergence result in $H^{3}(\Omega)$ also allows us to conclude that both the Dirichlet (on $\Gamma_{l}$ ) and the Neumann condition (on $\Gamma_{0}$ ) pass to the limit for $\varphi_{n}$. Using again the convergence of $\mathbb{P}_{\Psi_{n}}$ and the fact that $\psi_{i}$ satisfies the homogeneous Dirichlet and Neumann boundary conditions, we deduce that $\varphi$ satisfies (60). For $\mu$, we know that $\mu_{n}$ converges weakly to $\mu$ in $\Phi_{0}^{1}$.

It remains to prove that the functions $\boldsymbol{u}_{\varphi}, \varphi$ and $\mu$ satisfy (58), (59). Let $\rho \in \mathcal{D}^{\prime}\left(\mathbb{R}^{+}\right)$, and let $N>1$. For any $n \geqslant N, \varphi_{n}$ satisfies (58) with $\psi=\mu_{N}$. We multiply this equation by $\rho(t)$ and integrate by parts. From the convergence results stated above, we can pass to the limit in this equation. The limit equation obtained is fulfilled for any $N \geqslant 1$, and any $\rho \in \mathcal{D}^{\prime}\left(\mathbb{R}^{+}\right)$, thus we conclude from the density of $\operatorname{Span}\left(\psi_{i}\right)_{i \geqslant 1}$ in $H^{1}(\Omega)$ that $\boldsymbol{u}_{\varphi}, \varphi$ and $\mu$ satisfy (58), where $\boldsymbol{u}_{\varphi}$ is defined by the formulas (22)- $(23)$ and $(25)$.

Lastly, since $\mathbb{P}_{\Psi_{n}}$ converges to the identity for the strong topology of operators (see Remark 5.10), the dominated convergence theorem allows us to conclude that $\varphi$ and $\mu$ also satisfy $(59)$.

\section{Numerical illustration}

In this section, we present some preliminary numerical results solving system (26), in order to show some features of the model. Let us emphasize that in contrary to other bifluid models, this model does not assume that the interface between the two fluids is a graph, and therefore allows more general configurations, such as drops.

The equations are discretized in a standard way by finite differences. In order to deal with the fact that the domain is not rectangular, we rescaled the equations to work in the rescaled domain $\Omega_{\text {rescaled }}=\{(x, y), x \in(0, L), y \in$ $(0,1)\}$. In order to preserve a maximal principle on $\varphi$, we use the same flux limiters for the Cahn-Hilliard equation as in [5]. The boundary conditions are treated by introducing artificial variables in fictive cells on the boundary of the domain.

\subsection{Influence of the different viscosities}

Viscosity is widely used for fluid characterization, and allows us to model different types of behavior for the fluids, even for Newtonian ones (which is the framework of this study). It is of interest to compare the results obtained in both scenarios, when a drop of a less viscous fluid is immersed in a more viscous one, or when a drop of a more viscous fluid is immersed in a less viscous one. Indeed, the results can vary in a qualitative way.

In order to focus on the influence of the viscosity, we use a simple domain of constant thickness $h \equiv 1$, and we neglect the shear effects by choosing the shear velocity $s=0$. The test cases are carried out with the parameter $\alpha$ related to the thickness of the interface chosen equal to $\alpha=0.015$, with an input flow $Q=0.5$. The time step $\delta t$ is adapted from the C.F.L. condition, with $\delta t \leqslant 0.01$. Thus, we model a situation in which the flow "pushes" the drop in the other fluid, from the left hand side to the right. 
- If we want to model for example a drop of oil in water, we choose $\eta_{2} / \eta_{1}=$ 80. We obtain the results presented in Figure 5 . We observe that a viscous drop is not really deformed when immersed in a less viscous fluid.
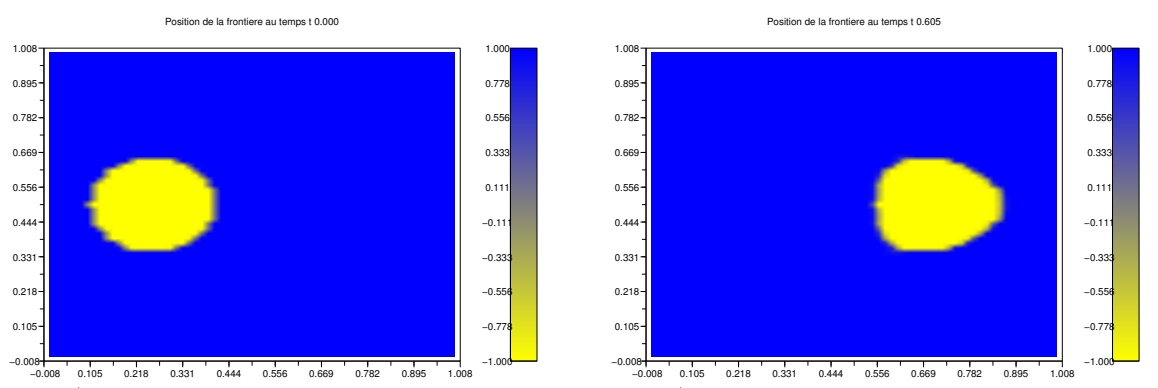

Figure 5: A drop of oil (in yellow) in water (in dark blue)

- On the other hand, choosing $\eta_{2} / \eta_{1}=1 / 80$, we model a drop of water in oil. The results are given in 6. On the contrary to the previous case, the drop is strongly deformed.
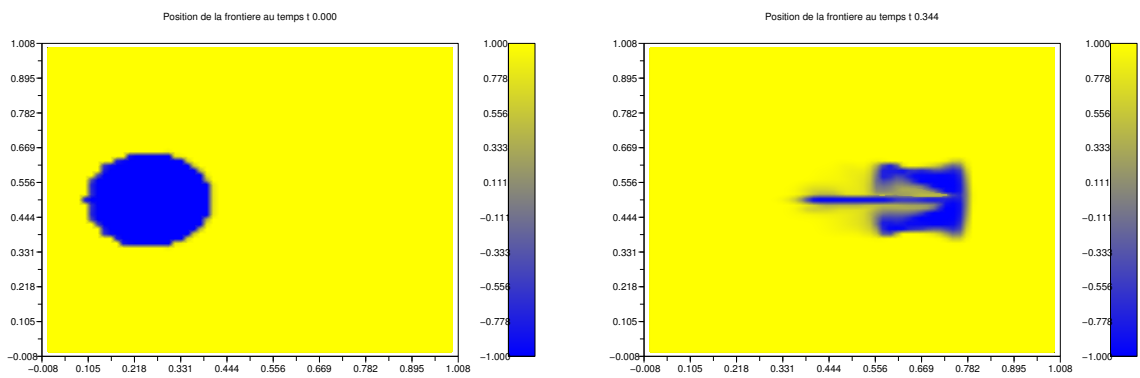

Figure 6: A drop of water (in dark blue) in oil (in yellow)

Of course, these numerical results could be enhanced with a model taking the surface tension into account.

\subsection{Drop transport applications}

Another example which allows to validate the program corresponds to the observation of recirculations inside a drop. Indeed, numerical and experimental works [11], 26] have showed that due to the blending dynamics, recirculations are observed.

If we compute the relative velocity, we observe recirculations inside the drops, as in Figure 77. To this end, we define a mean value of the velocity $\overline{\boldsymbol{u}}$, for example 

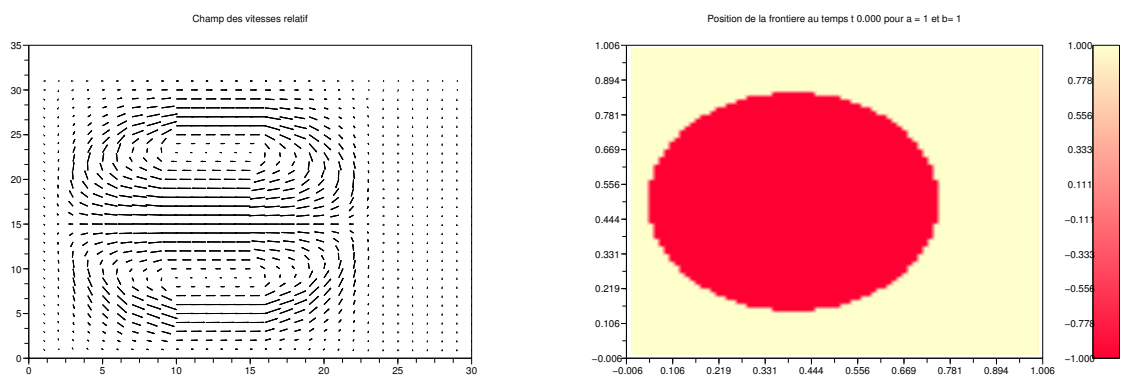

Figure 7: Recirculations in a drop and shape of the drop

the value on $\Gamma_{l}$ (outside the drop), and we compute $\boldsymbol{u}-\overline{\boldsymbol{u}}$, which is represented in the figure.

It is of interest to note that this asymptotic model, which is in fact a very simple one when comparing to the whole Navier-Stokes system coupled with the Cahn-Hilliard equation, allows us nevertheless to observe very fine phenomena, such as recirculations inside a drop.

\section{Appendix}

Proposition 7.1. Let $T>0$. Let $\mathcal{Y}$ and $\mathcal{Z}$ be two functions in $\mathcal{C}^{1}([0, T])$, such that there exists three real constants $C_{1}, C_{2}$ and a function $f: \mathbb{R} \rightarrow \mathbb{R}$ satisfying

$$
\mathcal{Y}^{\prime}+C_{1} \mathcal{Z} \leqslant f(\mathcal{Y}) \mathcal{Z}+C_{2}, \quad 0 \leqslant \mathcal{Y} \leqslant \mathcal{Z} \quad \text { on }[0, T]
$$

Assume that

- $f$ is an increasing continuous function such that $f(0)=0$,

- $C_{1}>0$,

- there exists $M>0$ such that

$$
f(M)<\frac{C_{1}}{2} \quad \text { and } \quad C_{2}<\frac{M C_{1}}{2} .
$$

If $\mathcal{Y}(0)<M$, then there exists a constant $C$ such that

$$
\|\mathcal{Y}\|_{L^{\infty}(0, T)} \leqslant M
$$

Moreover, we have

$$
\|\mathcal{Z}\|_{L^{1}(0, T)} \leqslant C T+C .
$$

Proof. Suppose that there exists $0<T^{*}<T$, such that $\mathcal{Y}\left(T^{*}\right)=M$ and $\mathcal{Y}^{\prime}\left(T^{*}\right)>0$. Then, evaluating (94) at $T^{*}$, and using the hypothesis on $C_{2}$, we get

$0<\mathcal{Y}^{\prime}\left(T^{*}\right) \leqslant \mathcal{Z}\left(T^{*}\right)\left(f(M)-C_{1}\right)+C_{2} \leqslant-\frac{C_{1}}{2} \mathcal{Z}\left(T^{*}\right)+C_{2} \leqslant \frac{C_{1}}{2}\left(M-\mathcal{Z}\left(T^{*}\right)\right)$. 
But since $M=\mathcal{Y}\left(T^{*}\right) \leqslant \mathcal{Z}\left(T^{*}\right)$, we have $M-\mathcal{Z}\left(T^{*}\right) \leqslant 0$, which leads to a contradiction.

The regularity of $\mathcal{Z}$ follows by integrating $(94)$ over $(0, T)$, and using the regularity of $\mathcal{Y}$ :

$$
\frac{C_{1}}{2}\|\mathcal{Z}(t)\|_{L^{1}(0, T)} \leqslant \mathcal{Y}(T)+\frac{C_{1}}{2}\|\mathcal{Z}(t)\|_{L^{1}(0, T)} \leqslant \mathcal{Y}(0)+C_{2} T \leqslant M+C_{2} T,
$$

which is written $\|\mathcal{Z}(t)\|_{L^{1}(0, T)} \leqslant C T+C$.

\section{Acknowledgments}

The authors want to thank the reviewers for their relevant and helpful remarks and comments, which helped to improve the paper.

\section{Bibliography}

[1] A. Assemien, G. Bayada, and M. Chambat, Inertial effects in the asymptotic behavior of a thin film flow, Asymptotic Anal., 9(3) (1994) 177-208.

[2] R.A. Adams, Sobolev spaces, Pure and Applied Mathematics 65 (Academic Press, New York-London, 1975).

[3] G. Bayada and M. Chambat, The transition between the Stokes equations and the Reynolds equation: a mathematical proof, Appl. Math. Optim., 14(1) (1986) 73-93.

[4] G. Bayada, M. Chambat, and I. Ciuperca, Asymptotic Navier-Stokes equations in a thin moving boundary domain, Asymptot. Anal., 21(2) (1999) $117-132$.

[5] F. Boyer, L. Chupin and P. Fabrie, Numerical study of viscoelastic mixtures through a Cahn-Hilliard flow model, Eur. J. Mech. B Fluids, 23(5) (2004) 759-780.

[6] H. Beirão da Veiga, On the $W^{2, p}$-regularity for solutions of mixed problems, J. Math. Pures Appl., 9(53) (1974) 279-290.

[7] G. Bayada, S. Martin and C. Vázquez, About a generalized BuckleyLeverett equation and lubrication multifluid flow, European J. Appl. Math., 17(5) (2006) 491-524.

[8] F. Boyer, Mathematical study of multi-phase flow under shear through order parameter formulation, Asymptot. Anal., 20(2) (1999) 175-212.

[9] F. Boyer, Nonhomogeneous Cahn-Hilliard fluids, Ann. Inst. H. Poincaré Anal. Non Linéaire, 18(2) (2001) 225-259.

[10] F. Boyer, A theoretical and numerical model for the study of incompressible mixture flows, Computers and Fluids, 31(1) (2002) 41-68. 
[11] T.Colin, G. Cristobal, C. Galusinski, K. Khadra and P. Vigneaux, Ecoulement de gouttes dans des microcanaux: simulations numériques et expériences, Proceedings du 18e congrès français de mécanique, Grenoble) (2007).

[12] L. Chupin, Existence result for a mixture of non Newtonian flows with stress diffusion using the Cahn-Hilliard formulation, Discrete Contin. Dyn. Syst. Ser. B, 3(1) (2003) 45-68.

[13] L. Chupin, Some theoretical results concerning diphasic viscoelastic flows of the Oldroyd kind, Adv. Differential Equations, 9(9-10) (2004) 1039-1078.

[14] L. Chupin and B. Grec, Steady state solutions for a lubrication two-fluid flow, Eur. J. Appl. Math., 22(6) (2011) 581-612.

[15] M. Dauge, Elliptic boundary value problems on corner domains, Smoothness and asymptotics of solutions, 1341 (Springer-Verlag, Berlin, 1988).

[16] M. Doi, Dynamics of domains and textures, in Theoretical Challenges in the Dynamics of Complex Fluids, (T.C.B. McLeish, 1997) 293-314.

[17] C. M. Elliott and H. Garcke, On the Cahn-Hilliard equation with degenerate mobility, SIAM J. Math. Anal., 27(2) (1996) 404-423.

[18] V. Girault and P.-A. Raviart, Finite element approximation of the NavierStokes equations, Lecture Notes in Mathematics, 749 (Springer-Verlag, Berlin, 1979).

[19] P. Grisvard, Elliptic problems in nonsmooth domains, Monographs and Studies in Mathematics, 24 (Pitman, Boston, 1985).

[20] J.D. Guton, M. San Miguel, and P.S. Sahni, Phase transitions and critical phenomena, 8 (Academic, London, 1983).

[21] L. Hörmander, Lectures on nonlinear hyperbolic differential equations, Mathématiques \& Applications, 26 (Springer-Verlag, Berlin, 1997).

[22] E. Marušić-Paloka and M. Starčević, Rigorous justification of the Reynolds equations for gas lubrication, C. R. Mécanique, 33(7) (2005) 534-541.

[23] A. Onuki, Phase transitions of fluids in shear flow, J. Phys. Condens. Matter, 9 (1997) 6119-6157.

[24] L. Paoli, Asymptotic behavior of a two fluid flow in a thin domain: from Stokes equations to Buckley-Leverett equation and Reynolds law, Asymptot. Anal., 34(2) (2003) 93-120.

[25] J. Simon, Compact sets in the space $L^{p}(0, T ; B)$, Ann. Mat. Pura Appl., 4(146) (1987) 65-96. 
[26] F. Sarrazin, K. Loubière, L. Prat, C. Gourdon, T. Bonometti and J. Magnaudet, Experimental and numerical study of droplets hydrodynamics in microchannels, AIChE Journal, 52(12) (2006) 4061-4070.

[27] R. Temam, Infinite-dimensional dynamical systems in mechanics and physics, Applied Mathematical Sciences, 68 (Springer-Verlag, New York, second edition, 1997). 